\title{
O Número de Nielsen Relativo
}

\author{
Claudemir Aniz
}

Orientador: Prof. Dr. OzIRIde Manzoli Neto

Dissertação apresentada ao Institudo de Ciências Matemáticas e de Computação-USP, como parte dos requisitos para obtenção do título de Mestre em Ciências - Área: Matemática.

\section{USP - Sāo Carlos}

Maio de 1998 
Ao meu pai Antonio Ismael Aniz (in memorian). 


\section{Agradecimentos}

Aos professores Oziride Manzoli Neto e Lucília Daruiz Borsari, que foram meus orientadores e meus amigos.

A minha mãe Aparecida de Abreu Aniz, meus irmãos José Antonio, Reginaldo e Ismael, pelo incentivo que em muitos momentos tornou-se indispensável.

A todos os meus professores, em particular para a professora Ires Dias pelo apoio e atenção.

Aos meus amigos André, Carlos e Alexandra, que foram colegas de estudo.

Ao CNPq, pelo auxílio financeiro. 


\section{Resumo}

O objetivo deste trabalho é introduzir o número de Nielsen relativo $N(f ; X, A)$, para aplicações $f:(X, A) \rightarrow(X, A)$ entre pares de espaços, com propriedades semelhantes aos do número de Nielsen, como invariância homotópica e invariância por tipo de homotopia. De $N(f ; X, A) \geq N(f)=$ $N(f ; X, \emptyset)$, o número de Nielsen relativo é no caso $A \neq \emptyset$ um limitante inferior melhor do que $N(f)$ para o número mínimo $\mu(f ; X, A)$ de pontos fixos na classe de homotopia de $f$, onde as homotopias são aplicações da forma $H:(X \times I, A \times I) \rightarrow(X, A)$. Condições para um par $(X, A)$ de poliedros finitos são dadas para assegurar que o número de Nielsen relativo é de fato o melhor limitante inferior, isto é, $N(f ; X, A)=\mu(f ; X, A)$. 


\begin{abstract}
The purpose of this work is to introduce the relative Nielsen number $N(f ; X, A)$ for maps of pairs of spaces $f:(X, A) \rightarrow(X, A)$, with similar properties to the usual Nielsen number as homotopy invariance and homotopy type invariance. From $N(f ; X, A) \geq N(f)=N(f ; X, \emptyset)$, the relative Nielsen number is in the case $A \neq \emptyset$ a better lower bound than $N(f)$ for the minimum number $\mu(f ; X, A)$ of fixed points in the homotopy class of $f$, here homotopy means maps of pairs of the form $H:(X \times I, A \times I) \rightarrow(X, A)$. In the case $(X, A)$ is a finite polyhedral pair, conditions are given to guarantee that the relative Nielsen number is in fact the best lower bound, that is, $N(f ; X, A)=\mu(f ; X, A)$.
\end{abstract}




\section{Índice}

$\begin{array}{ll}\text { Introduçāo } & 1\end{array}$

1 Preliminares 4

1.1 Teoria de Índice para Poliedros . . . . . . . . . . . . . 4

1.2 O Número de Nielsen . . . . . . . . . . . . . . . . . 11

1.3 Teorema de Minimização . . . . . . . . . . . . . . 17

2 O Número de Nielsen Relativo 22

2.1 Definição e Propriedades . . . . . . . . . . . . . . . . 22

2.2 Teorema de Minimização Relativo . . . . . . . . . . . . . 29

Apêndice $\quad 41$

$\begin{array}{ll}\text { Referências Bibliográficas } & \cdot 51\end{array}$ 


\section{Introdução}

A teoria de ponto fixo de Nielsen preocupa-se com a determinação do número mínimo $\mu(f)$ de pontos fixos na classe de homotopia de uma dada aplicação $f: X \rightarrow X$ (isto é, uma função contínua). Para este propósito o número de Nielsen $N(f)$ é introduzido, que é sempre um limitante inferior para $\mu(f)$ e em muitos casos o melhor limitante inferior. No entanto, se $f:(X, A) \rightarrow(X, A)$ é uma auto aplicação de pares de espaço e as homotopias são aplicações da forma $H:(X \times I, A \times I) \rightarrow(X, A)$, percebemos que $N(f)$ é um mau limitante inferior para o número mínimo $\mu(f ; X, A)$ de pontos fixos na classe de homotopia de $f:(X, A) \rightarrow(X, A)$. Para ver isto, considere o caso onde $X=B^{2}$ é um disco e $A=S^{1}$ o círculo que o borda. Se $f:\left(B^{2}, S^{1}\right) \rightarrow\left(B^{2}, S^{1}\right)$ é uma aplicação onde $\left.f\right|_{S^{1}}$ tem grau $d$, então todas as aplicações homotópicas à $f:\left(B^{2}, S^{1}\right) \rightarrow\left(B^{2}, S^{1}\right)$ tem pelo menos $|d-1|$ pontos fixos sobre $S^{1}$ (ver exemplo 1.2.1). Portanto $|d-1| \leq \mu(f ; X, A)$, mas $N(f)=1$. Daí a necessidade de introduzir um número de Nielsen "relativo"para aplicações de pares de espaços, que faz o mesmo papel de $N(f)$ e é um melhor limitante inferior para $\mu(f ; X, A)$.

O capítulo um, usa como referências [8], [5], [6] e [3]. Neste capítulo apresentamos de forma resumida a teoria de ponto fixo de Nielsen. Definimos índice para poliedros finitos conexos, listamos suas principais propriedades e calculamos índice de pontos fixos isolados de funções reais. A definição do 
número de Nielsen e as demonstrações de suas propriedades básicas é feita na secção 1.2 .

Na seç̧ão 1.3 descrevemos o problema de determinar o número mínimo de pontos fixos, enunciamos o Teorema de Minimização 1.3.1 e apresentamos alguns resultados técnicos indispensáveis para o desenvolvimento do capítulo 2 .

O propósito do capítulo dois, que é baseado em Schirmer [1] é introduzir o número de Nielsen relativo $N(f ; X, A)$. A definição de $N(f ; X, A)$ na seç̧ão 2.1 usa a definição existente de classe de pontos fixos para uma aplicação $f: X \rightarrow X$. Mais precisamente, $N(f ; X, A)$ é obtido adicionando ao número $N(f)$ de classes de ponto fixos essenciais de $f: X \rightarrow X$, isto é, a aplicação $f$ considerada como uma auto aplicação de $X$ somente, o número $N(\bar{f})$ de classes de ponto fixo essenciais da restrição $\bar{f}: A \rightarrow A$ de $f$ para $A$, e então subtraindo o número $N(f, \bar{f})$ de classes essenciais "comuns" de $f$ e $\bar{f}$, onde uma classe comum de $f$ e $\bar{f}$ é definida como uma classe de ponto fixo de $f$ que intercepta uma classe de ponto fixo essencial de $\bar{f}$. A definição de $N(f ; X, A)$ produz um inteiro positivo que tem as propriedades básicas usuais do número de Nielsen $N(f)$. Este é invariante por homotopia, invariante por tipo de homotopia, e um limitante inferior de $\mu(f ; X, A)$.

$\mathrm{Na}$ secção 2.2 consideramos a questão de quando $N(f ; X, A)$ é de fato o melhor limitante inferior para $\mu(f ; X, A)$, isto é, quando existe uma aplicação $g:(X, A) \rightarrow(X, A)$ homotópica a aplicação $f:(X, A) \rightarrow(X, A)$ que tem precisamente $N(f ; X, A)$ pontos fixos. O Teorema de Minimização Relativo 2.2.4 mostra que este é o caso quando supomos $(X, A)$ satisfazendo propriedades bastante gerais. A construção de uma aplicação com um conjunto de pontos fixos mínimo se dá, como usual, em dois passos. Primeiro deformamos $f$ a uma aplicação que tem apenas um número finito de pontos 
fixos e tomamos o cuidado de assegurar que esta aplicação tem somente $N(\bar{f})$ pontos fixos sobre $A$ (teorema 2.2.2). Depois unimos pontos fixos em $\overline{X-A}$ quando possível.

As hipóteses do Teorema de Minimização Relativo devem por necessidade incluir aquelas que são necessárias se $A=\emptyset$ ou $A=X$, mas isto não é suficiente. A nova hipótese que surge em nossa situação é que $A$ pode ser "by-passed"em $X$, isto é, todo caminho em $X$ com pontos finais em $X-A$ pode ser deformado a um longe de $A$. O problema de determinar $\mu(f ; X, A)$ quando não temos como hipótese $A$ "by-passed"foi estudado por Zhao [4], onde um novo tipo de número de Nielsen $m(f ; X, A)$ é definido, e $N(f ; X, A) \leq m(f ; X, A)$.

Para demonstrar o Teorema de Minimização Relativo usamos o lema 2.2.6 desenvolvido por Boju Jiang [2] que tem como hipótese, $X=|K|$ um poliedro finito conexo sem ponto de corte local. No teorema original de Shirmer tinhamos apenas $X-A$ sem ponto de corte local, e foi preciso substitui-la, para que o lema pudesse ser aplicado.

O apêndice usa como referência [2] e contém técnicas para obter arcos PL-normais além da demonstração do lema 2.2.6. 


\section{Capítulo 1}

\section{Preliminares}

\subsection{Teoria de Índice para Poliedros}

Seja $X$ um espaço topológico. Um ponto fixo de uma aplicação $f: X \rightarrow X$ é um ponto $x \in X$ tal que $f(x)=x$.

O conjunto dos pontos fixos de uma aplicação $f$ será denotado por $\operatorname{Fix}(f)$.

Seja $V \subset \mathbb{R}^{n}$ um subconjunto aberto e $g: V \rightarrow \mathbb{R}^{n}$ uma aplicação contínua tal que $\operatorname{Fix}(g)$ é compacto. Considere a composta

$$
\begin{gathered}
H_{n}\left(\mathbb{R}^{n}, \mathbb{R}^{n}-0\right) \stackrel{\left(j_{1_{*}}\right)^{-1}}{\longrightarrow} H_{n}\left(\mathbb{R}^{n}, \mathbb{R}^{n}-D\right) \stackrel{j_{2 *}}{\longrightarrow} H_{n}\left(\mathbb{R}^{n}, \mathbb{R}^{n}-\operatorname{Fix}(g)\right) \\
\stackrel{(e x c)^{-1}}{\longrightarrow} H_{n}(V, V-\operatorname{Fix}(g)) \stackrel{(i-g) \cdot}{\longrightarrow} H_{n}\left(\mathbb{R}^{n}, \mathbb{R}^{n}-0\right),
\end{gathered}
$$

on de $j_{1}$ e $j_{2}$ são inclusões, $(i-g)$ é a aplicação $(i-g)(x)=x-g(x)$ e $D$ uma bola fechada em torno da origem contendo $\operatorname{Fix}(g)$. Como $\left(j_{1}\right)_{*}$ é isomorfismo, a composta está bem definida.

O fato de $H_{n}\left(\mathbb{R}^{n}, \mathbb{R}^{n}-0\right) \cong \mathbb{Z}$ diz que a composta acima é um endomorfismo de $\mathbb{Z}$. Logo tem a forma

$$
(i-g)_{*} \circ(e x c)^{-1} \circ\left(j_{2_{*}}\right) \circ\left(j_{1_{*}}\right)^{-1} .(x)=I\left(\mathbb{R}^{n}, g, V\right) \cdot x
$$


onde $I\left(\mathbb{R}^{n}, g, V\right)$ é o único inteiro que determina o endomorfismo dada pela composta.

Definiçāo 1.1.1 Usando as hipóteses acima, $I\left(\mathbb{R}^{n}, g, V\right)$ é chamado o índice dos pontos fixos de $g$.

Veremos agora como se calcula o índice de um ponto fixo isolado de uma função real.

Exemplo 1.1.1 Seja $g: \mathbb{R} \rightarrow \mathbb{R}$ uma função contínua tal que $\operatorname{Fix}(g) \cap V=$ $\{0\}$, onde $V=(-\varepsilon, \varepsilon)$ com $\varepsilon>0$. O índice $I(\mathbb{R}, g, V)$ é obtido da composta

$$
\begin{gathered}
H_{1}(\mathbb{R}, \mathbb{R}-0) \stackrel{\left(j_{1_{*}}\right)^{-1}}{\longrightarrow} H_{1}(\mathbb{R}, \mathbb{R}-\bar{V}) \stackrel{j_{2} .}{\longrightarrow} H_{1}(\mathbb{R}, \mathbb{R}-0) \\
\stackrel{(\text { exc })^{-1}}{\longrightarrow} H_{1}(V, V-0) \stackrel{(i-g)_{*}}{\longrightarrow} H_{1}(\mathbb{R}, \mathbb{R}-0)
\end{gathered}
$$

Como neste caso $j_{2_{*}} \circ\left(j_{1_{*}}\right)^{-1}=i d$, temos somente que calcular a composta

$$
H_{1}(\mathbb{R}, \mathbb{R}-0) \stackrel{(e x c)^{-1}}{\longrightarrow} H_{1}(V, V-0) \stackrel{(i-g) \cdot}{\longrightarrow} H_{1}(\mathbb{R}, \mathbb{R}-0)
$$

Como o diagrama

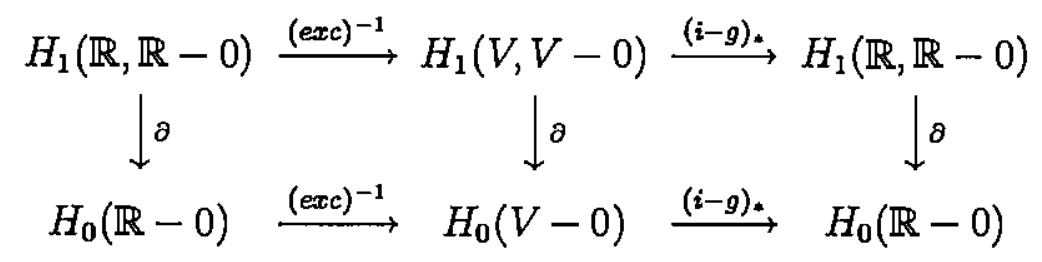

é comutativo, temos que

$$
\partial \circ(i-g)_{*} \circ(e x c)^{-1}=(i-g)_{*} \circ(e x c)^{-1} \circ \partial
$$

Seja $a \in H_{1}(\mathbb{R}, \mathbb{R}-0) \cong \mathbb{Z}$ e $b, c \in H_{0}(\mathbb{R}-0) \cong \mathbb{Z} \oplus \mathbb{Z}$ geradores.

Sem perda de generalidade podemos supor que $\partial(a)=b-c$ (caso contrário $\partial(a)=c-b)$.

Para determinar a composta é suficiente analisar o comportamento de 
$\left.(i-g)\right|_{(V-0)}$ e observar que as $\partial$ 's são injetoras.

Temos três casos:

Caso 1: $\operatorname{Im}(i-g)$ está contida em uma componente de $\mathbb{R}-0$.

Caso 2: $\operatorname{Im}(i-g)$ preserva as componentes.

Caso 3: $\operatorname{Im}(i-g)$ reverte as componentes.

Em homologia temos:

Caso 1: A imagem de $\partial(a)$ é levada em zero, pois;

$$
\partial \circ(i-g)_{*} \circ(e x c)^{-1}(a)=(i-g)_{*} \circ(e x c)^{-1} \circ \partial(a)=0,
$$

portanto $(i-g) *(e x c)^{-1}(a)=0$ e o índice é zero.

Caso 2: A imagem de $\partial(a)$ é levada nele próprio, pois;

$$
\partial \circ(i-g)_{*} \circ(e x c)^{-1}(a)=(i-g)_{*} \circ(e x c)^{-1} \circ \partial(a)=b-c,
$$

e temos $(i-g)_{*} \circ(e x c)^{-1}(a)=a$, logo o índice é 1.

Caso 3: A imagem de $\partial(a)$ é levada em $-b+c$, pois;

$$
\partial \circ(i-g) *(e x c)^{-1}(a)=(i-g)_{*} \circ(e x c)^{-1} \circ \partial(a)=-b+c,
$$

segue que $(i-g)_{*} \circ(e x c)^{-1}(a)=-a$, logo o índice é -1 . 
O gráfico de $g$ em cada caso é:

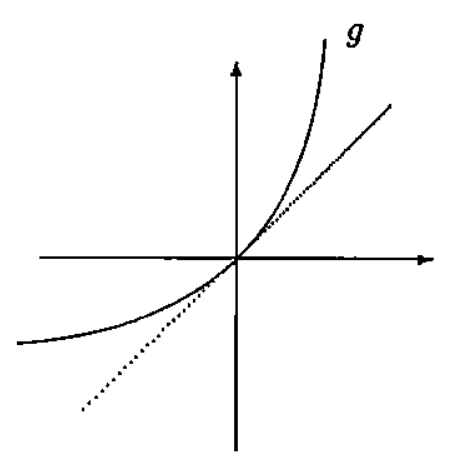

caso 1
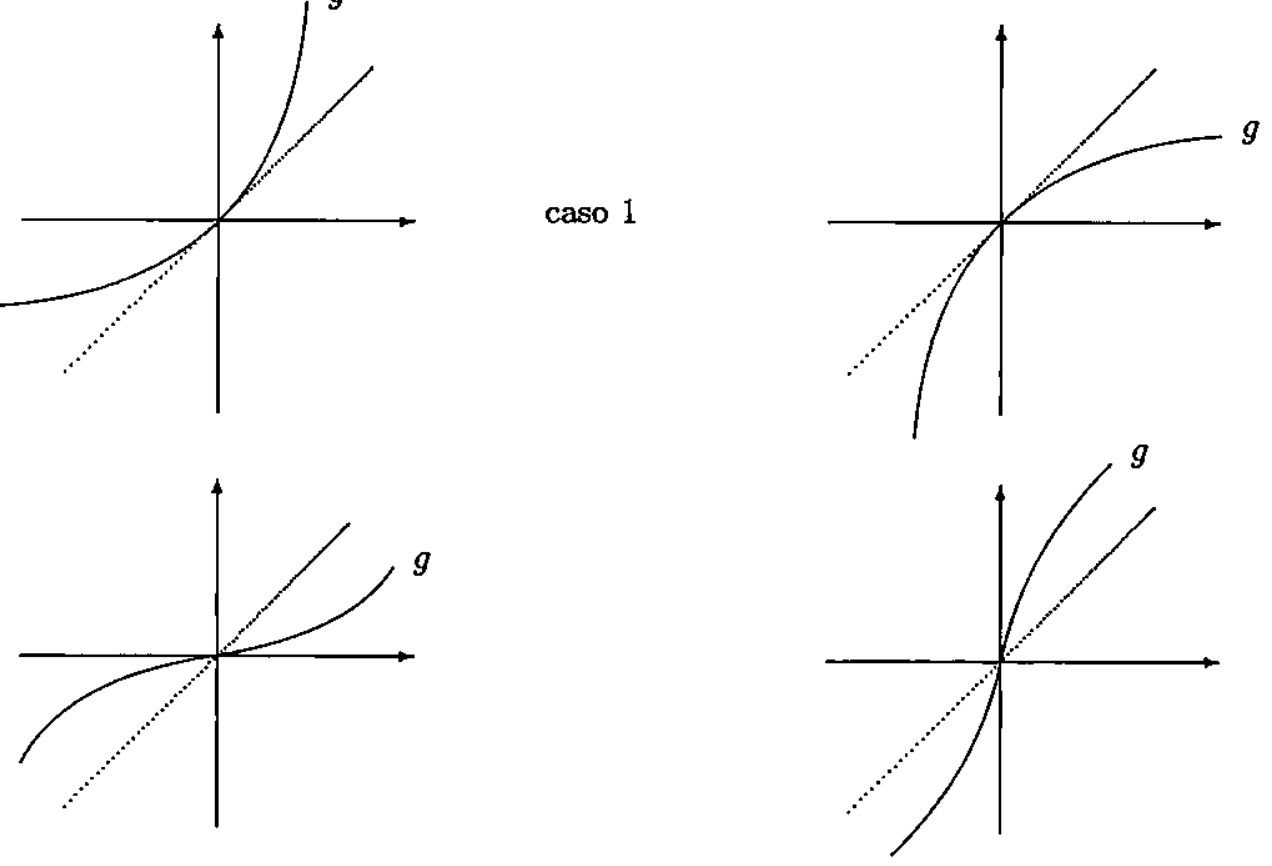

caso 2

caso 3

\section{Figura 1.1:}

Note que os gráficos de $g$ satisfazem:

Caso 1: $-\varepsilon<g(-\varepsilon)$ e $\varepsilon<g(\varepsilon)$

$$
-\varepsilon>g(-\varepsilon) \text { e } \varepsilon>g(\varepsilon)
$$

Caso 2: $-\varepsilon<g(-\varepsilon)$ e $\varepsilon>g(\varepsilon)$

Caso 3: $-\varepsilon>g(-\varepsilon)$ e $\varepsilon<g(\varepsilon)$

Este exemplo pode ser generalizado para qualquer ponto fixo isolado.

Suponha que $x_{0}$ é um ponto fixo isolado de $f: \mathbb{R} \rightarrow \mathbb{R}$, i.é, existe um número positivo $\varepsilon$ tal que $f$ tem somente o ponto fixo $x_{0}$ sobre o intervalo fechado $\bar{V}=\left[x_{0}-\varepsilon, x_{0}+\varepsilon\right]$. Existem exatamente 4 casos: 
Caso 1: $x_{0}-\varepsilon<f\left(x_{0}-\varepsilon\right)$ e $x_{0}+\varepsilon>f\left(x_{0}+\varepsilon\right)$

Caso 2: $x_{0}-\varepsilon>f\left(x_{0}-\varepsilon\right)$ e $x_{0}+\varepsilon<f\left(x_{0}+\varepsilon\right)$

Caso 3: $x_{0}-\varepsilon<f\left(x_{0}-\varepsilon\right)$ e $x_{0}+\varepsilon<f\left(x_{0}+\varepsilon\right)$

Caso 4: $x_{0}-\varepsilon>f\left(x_{0}-\varepsilon\right)$ e $x_{0}+\varepsilon>f\left(x_{0}+\varepsilon\right)$

Daí

$$
I(\mathbb{R}, f, V)= \begin{cases}1 & \text { caso } 1 \\ -1 & \text { caso } 2 \\ 0 & \text { caso } 3 \text { e } 4\end{cases}
$$

O resultado a seguir será importante para a extensão do conceito de índice para uma classe mais geral do que os espaços Euclidianos, a saber o conjunto dos poliedros finitos conexos.

Teorema 1.1.1 (Comutatividade) Sejam $U \subset \mathbb{R}^{n}, U^{\prime} \subset \mathbb{R}^{n^{\prime}}$ dois abertos e $f: U \rightarrow \mathbb{R}^{n^{\prime}}, g: U^{\prime} \rightarrow \mathbb{R}^{n}$ aplicações contínuas. Considere $g \circ f: V=$ $f^{-1}\left(U^{\prime}\right) \rightarrow \mathbb{R}^{n}$ e $f \circ g: V^{\prime}=g^{-1}(U) \rightarrow \mathbb{R}^{n^{\prime}}$. Então $\operatorname{Fix}(f \circ g)$ é homeomorfo à $\operatorname{Fix}(g \circ f)$ e caso estes conjuntos forem compactos temos,

$$
I\left(\mathbb{R}^{n}, g \circ f, V\right)=I\left(\mathbb{R}^{n^{\prime}}, f \circ g, V^{\prime}\right)
$$

Prova: Veja [8], pág 34.

Vamos agora estender o conceito de índice para a categoria dos poliedros finitos conexos, que denotaremos por $C_{P}$.

Definiçāo 1.1.2 Uma terna $(X, f, U)$ é dita $C_{P}$-admissível se $X \in C_{P}$, $f: X \rightarrow X$ é uma aplicação, $U \subseteq X$ é um aberto de $X$ e f não tem ponto fixo no bordo de $U$.

O símbolo $C_{P}^{\prime}$ será usado para denotar a coleção de todas as ternas $C_{P^{-}}$ admissíveis. 
Dado $(X, f, U) \in C_{P}^{\prime}$, existe para algum $n$ um retrato de vizinhança $Y \subset \mathbb{R}^{n}$, homeomorfo a $U$. Observe que podemos decompor $f: U \rightarrow X$ na forma

$$
U \stackrel{i}{\longrightarrow} V \stackrel{f \circ r}{\longrightarrow} X
$$

onde $i$ é o homeomorfismo entre $U$ e $Y$ com $Y \subset V \subseteq \mathbb{R}^{n}, V$ aberto e $r$ a retração de $V$ em $U$, isto é; $r \circ i=i d$. Então colocando-se $\alpha=i$ e $\beta=f \circ r$ tem-se $f=\beta \circ \alpha$.

Como $\operatorname{Fix}(\alpha \circ \beta)$ é homeomorfo à $\operatorname{Fix}(\beta \circ \alpha)=\operatorname{Fix}\left(\left.f\right|_{U}\right)$ e $f$ não tem pontos fixos sobre o bordo de $U$, temos $\operatorname{Fix}\left(f_{\mid U}\right)$ compacto e $I\left(\mathbb{R}^{n}, \alpha \circ \beta, \beta^{-1}(U)\right)$ pode ser definido.

Definiçāo 1.1.3 Para cada terna $(X, f, U) \in C_{P}^{\prime}$ podemos associar um inteiro $i(X, f, U)$ chamado índice dos pontos fixos de $f$ em $U$ pela fórmula

$$
i(X, f, U)=I\left(\mathbb{R}^{n}, \alpha \circ \beta, \beta^{-1}(U)\right)
$$

Note que $i(X, f, U)$ está bem definido, pois caso $f: U \rightarrow X$ pudesse ser fatorado como

$$
U \stackrel{\gamma}{\longrightarrow} V^{\prime} \stackrel{\lambda}{\longrightarrow} X,
$$

onde $V^{\prime}$ é um aberto de $\mathbb{R}^{n^{\prime}}$, considerando as aplicações

$$
\gamma \circ r: V \rightarrow V^{\prime} \subset \mathbb{R}^{n^{\prime}}, i \circ \lambda: \lambda^{-1}(U) \rightarrow V \subset \mathbb{R}^{n}
$$

obteremos, pela comutatividade, aplicações

$$
\begin{aligned}
& (\gamma \circ r) \circ(i \circ \lambda)=\gamma \circ(r \circ i) \circ \lambda=\gamma \circ \lambda \\
& (i \circ \lambda) \circ(\gamma \circ r)=i \circ(\lambda \circ \gamma) \circ r=i \circ f \circ r
\end{aligned}
$$

com mesmo índice, isto é, $I\left(\mathbb{R}^{n^{\prime}}, \gamma \circ \lambda, \lambda^{-1}(U)\right)=I\left(\mathbb{R}^{n}, i \circ f \circ r,(f \circ r)^{-1}(U)\right)$, como o lado direito não depende da fatoração de $f$, tem-se que $i(X, f, U)$ é independente da fatoração de $f$. 
Exemplo 1.1.2 Vemos que para $X=S^{1}$ e $z_{0}$ é um ponto fixo isolado de uma auto aplicação $f$, isto é, existe $U \subset S^{1}$ aberto tal que $\operatorname{Fix}(f) \cap U=\left\{z_{0}\right\}$, é fácil calcular $i(X, f, U)$. Existe um homeomorfismo $i: U \rightarrow(-\varepsilon, \varepsilon)$ que leva $z_{0}$ em 0 , sendo assim $f$ pode ser decomposta na forma

$$
U \stackrel{i}{\rightarrow}(-\varepsilon, \varepsilon) \stackrel{i^{-1}}{\rightarrow} U \stackrel{f}{\rightarrow} S^{1}
$$

colocando-se $\beta=f \circ i^{-\mathbf{1}}$, tem-se por definição

$$
i\left(S^{1}, f, U\right)=I\left(\mathbb{R}, i \circ \beta, \beta^{-1}(U)\right) .
$$

Como $i \circ \beta: \beta^{-1}(U) \rightarrow \mathbb{R}$ tem como único ponto fixo $x=0$, recaímos no problema de calcular índice de ponto fixo isolado de uma função real. Considerando $f_{n}: S^{1} \rightarrow S^{1}$, onde $f(z)=z^{n} ;$ temos que se $n>1$ o índice de cada ponto fixo é -1 e se $n<1$ o índice é +1 .

O índice de pontos fixos satisfaz as seguintes propriedades, cujas demonstrações podem ser encontradas em [5], Cap.IV.

\section{Localizaçāo}

Se $(X, f, U) \in C_{P}^{\prime}$ e $g: X \rightarrow X$ é uma aplicação tal que $g(x)=f(x)$ para todo $x \in \bar{U}$ (fecho de $U$ ) então

$$
i(X, f, U)=i(X, g, U)
$$

\section{Invariância Homotópica}

Para $X \in C_{P}$ e $H: X \times I \rightarrow X$ uma homotopia, defina $f_{t}: X \rightarrow X$ por $f_{t}(x)=H(t, x)$. Se $\left(X, f_{t}, U\right) \in C_{P}^{\prime}$ para todo $t \in I$, então

$$
i\left(X, f_{0}, U\right)=i\left(X, f_{1}, U\right)
$$

\section{Aditividade}

Se $(X, f, U) \in C_{P}^{\prime}$ e $U_{1}, \ldots, U_{s}$ é um conjunto de subconjuntos abertos mutuamente disjuntos de $U$ tal que $f(x) \neq x$ para todo $x \in\left[U-\bigcup_{j=1}^{s} U_{j}\right]$, 
então

$$
i(X, f, U)=\sum_{j=1}^{s} i\left(X, f, U_{j}\right)
$$

\section{Normalizaçāo}

Se $X \in C_{P}$ e $f: X \rightarrow X$ é uma aplicação, então

$$
\begin{gathered}
i(X, f, X)=\text { o número de Lefschetz } L(f) \text { de } f, \\
L(f)=\sum_{q=0}^{\infty}(-1)^{g} \operatorname{tr}\left(f_{g}\right),
\end{gathered}
$$

onde $\operatorname{tr}\left(f_{q}\right)$ é o traço do homomorfismo $f_{q}: H_{q}(X, \mathbb{Q}) \rightarrow H_{q}(X, \mathbb{Q})$ induzido pela $f$ nos grupos de homologia de $X$ com coeficientes racionais.

\section{Comutatividade}

Se $X, Y \in C_{P}$ e $f: X \rightarrow Y, g: Y \rightarrow X$ são aplicações tal que $(X, g \circ f, U) \in$ $C_{P}^{\prime}$, então

$$
i(X, g \circ f, U)=i\left(Y, f \circ g, g^{-1}(U)\right)
$$

\subsection{O Número de Nielsen}

Um caminho $\alpha$ em um espaço $X$ é uma aplicação contínua $\alpha: I \rightarrow X$, onde $I$ denota o intervalo $[0,1]$. Dado um caminho $\alpha$ em $X$, defina o caminho $\alpha^{-1}: I \rightarrow X$ por $\alpha^{-1}(t)=\alpha(1-t)$ para todo $t \in I$.

Para caminhos $\alpha, \beta: I \rightarrow X$ com $\alpha(1)=\beta(0)$, podemos formar um novo caminho $\alpha \beta$ por

$$
\alpha \beta= \begin{cases}\alpha(2 t) & \text { se } 0 \leq t \leq 1 / 2 \\ \beta(2 t-1) & \text { se } 1 / 2 \leq t \leq 1\end{cases}
$$


Dois caminhos $\alpha, \beta: I \rightarrow X$ são ditos homotópicos com pontos extremos fixos se existe uma aplicação contínua $\psi: I \times I \rightarrow X$ tal que

$$
\begin{array}{ll}
\psi(s, 0)=\alpha(0)=\beta(0) & \text { para todo } s \in I \\
\psi(s, 1)=\alpha(1)=\beta(1) & \text { para todo } s \in I \\
\psi(0, t)=\alpha(t) & \text { para todo } t \in I \\
\psi(1, t)=\beta(t) & \text { para todo } t \in I
\end{array}
$$

Notação: $\alpha \simeq \beta \operatorname{rel}\{0,1\}$.

Seja X um poliedro finito e $f: X \rightarrow X$ uma aplicação; dizemos que pontos fixos $x_{0}$ e $x_{1}$ de $f$ são f-equivalentes se existe um caminho $\alpha: I \rightarrow X$ com $\alpha(0)=x_{0}, \alpha(1)=x_{1}$, tal que $f \circ \alpha \simeq \alpha \operatorname{rel}\{0,1\}$. É fácil ver que a relação de $f$-equivalência é uma relação de equivalência sobre $\operatorname{Fix}(f)$. As classes de equivalência são chamadas classes de ponto fixo de $f$.

Teorema 1.2.1 Em um poliedro finito $X$ com métrica $d$, dado $\varepsilon>0$, existe $\delta>0$ tal que se

$$
W=\{(x, y) \in X \times X \mid d(x, y)<\delta\}
$$

então existe uma aplicação $\gamma: W \times I \rightarrow X$ tal que

$$
\begin{gathered}
\gamma(x, y, 0)=x, \gamma(x, y, 1)=y \\
\gamma(x, x, t)=x \text { para todo } t \in I \\
\operatorname{diam}(\gamma((x, y) \times I))<\varepsilon \text { para todo }(x, y) \in W
\end{gathered}
$$

Prova: Veja [5], pág 39.

Duas aplicações $f, g: X \rightarrow X$ são ditas $\varepsilon$-homotópicas, para $\varepsilon>0$, se existe uma aplicação $H: X \times I \rightarrow X$ tal que

$$
H(x, 0)=f(x) \text { para todo } x \in X
$$




$$
\begin{gathered}
H(x, 1)=g(x) \text { para todo } x \in X \\
\operatorname{diam}(H(x \times I))<\varepsilon \text { para qualquer } x \in X
\end{gathered}
$$

Corolário 1.2.1 Seja $X$ um poliedro finito e $\varepsilon>0$ dado. Existe $\delta>0$ tal que se $f, g: X \rightarrow X$ são aplicações e $d(f(x), g(x))<\delta$, para todo $x \in X$, então $f$ e $g$ são $\varepsilon$ - homotópicas.

Prova: Basta colocar $H(x, t)=\gamma(f(x), g(x), t)$

Teorema 1.2.2 Uma aplicação $f: X \rightarrow X$ sobre um poliedro finito tem um número finito de classes de ponto fixo.

Prova: Pelo teorema 1.2.1 existe $\delta>0$ tal que, se $W_{1}=\{(x, y) \in$ $X \times X \mid d(x, y)<\delta\}$, então existe uma aplicação $\gamma_{1}: W_{1} \times I \rightarrow X$ tal que $\gamma_{1}(x, y, 0)=x, \gamma_{1}(x, y, 1)=y$, e $\gamma_{1}(x, x, t)=x$ para todo $t \in I$. Pela continuidade uniforme de $f$, dado $\delta>0$, existe $\zeta>0, \zeta<\delta / 2$, tal que se $x, z \in X$ e $d(x, z)<\zeta$, então $d(f(x), f(z))<\delta / 2$. Pelo teorema 1.2.1, existe $\eta>0$ tal que se $W_{0}=\{(x, y) \in X \times X \mid d(x, y)<\eta\}$, então existe uma aplicação $\gamma_{0}: W_{0} \times I \rightarrow X$ com as propriedades: $\gamma_{0}(x, y, 0)=x$, $\gamma_{0}(x, y, 1)=y, \gamma_{0}(x, x, t)=x, \mathrm{e} \operatorname{diam}\left(\gamma_{0}((x, y) \times I)\right)<\zeta$. Agora suponha $x, y \in \operatorname{Fix}(f)$ e $d(x, y)<\eta$; então $\alpha(t)=\gamma_{0}(x, y, t)$ é um caminho de $x$ para y tal que $\operatorname{diam}(\alpha(I))<\zeta<\delta / 2$ e daí $\operatorname{diam}(f \circ \alpha(I))<\delta / 2$. Portanto, para $t \in I, d(\alpha(t), f \circ \alpha(t))<\delta$ e nós temos uma aplicação $H: I \times I \rightarrow X$ dada por $H(s, t)=\gamma_{1}(\alpha(s), f \circ \alpha(s), t)$ que mostra que $x, y$ são $f$-equivalentes. Nós então provamos que cada classe de ponto fixo é aberto em $\operatorname{Fix}(f)$. Como $\operatorname{Fix}(f)$ é compacto (pois é fechado dentro de um compacto), temos um número finito de classes de ponto fixos.

Seja $X$ um poliedro finito conexo e $f: X \rightarrow X$ uma aplicação. Para cada classe de ponto fixo $F$ da aplicação $f$ existe pelo teorema acima um aberto 
$U$ em $X$ tal que $F \subseteq U$ e $\bar{U} \cap \operatorname{Fix}(f)=F$. Note que $(X, f, U) \in C_{P}^{\prime}$. Defina o índice $i(F)$ da classe de ponto fixo $F$ por $i(F)=i(X, f, U)$.

Teorema 1.2.3 A definição de $i(F)$ é independente da escolha do conjunto aberto $U \subseteq X$ tal que $F \subseteq U$ e $\bar{U} \cap \operatorname{Fix}(f)=F$.

Prova: Seja $U$ e $V$ subconjuntos abertos de $\mathrm{X}$ satisfazendo as hipóteses do teorema. Se $x \in U-(U \cap V)$ então $x$ não pertence a nenhuma outra classe de ponto fixo de $f$. Como $x \notin V$, temos que $x \notin F$, daí $x \notin \operatorname{Fix}(f)$. Pela aditividade $i(X, f, U)=i(X, f, U \cap V)$. Usando o mesmo argumento temos que $i(X, f, V)=i(X, f, U \cap V)$. Portanto $i(X, f, U)=i(X, f, V)$.

Para $X$ um poliedro finito conexo e $f: X \rightarrow X$ uma aplicação, uma classe de ponto fixo $F$ de $f$ é dita essencial se $i(F) \neq 0$ e inessencial se $i(F)=0$. O número de Nielsen $N(f)$ da aplicação $f$ é definido sendo o número de classes de ponto fixo de $f$ que são essenciais.

Exemplo 1.2.1 Se uma auto aplicação $f: S^{1} \rightarrow S^{1}$ tem grau $n$. Então $N(f)=|1-n|(\operatorname{ver}[3]$, pág 33).

Exemplo 1.2.2 Seja $T^{n}=S^{1} \times \ldots \times S^{1}$ e $f: T^{n} \rightarrow T^{n}$ uma auto aplicação.

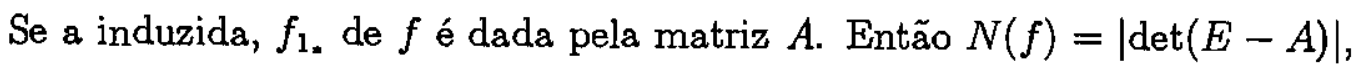
onde $E$ é a matriz identidade (ver [3], pág 33).

Teorema 1.2.4 Uma aplicação $f: X \rightarrow X$ sobre um poliedro finito conexo tem pelo menos $N(f)$ pontos fixos.

Sejam $X$ e $Y$ poliedros finitos, $H: X \times I \rightarrow Y$ uma aplicação contínua e $\alpha$ um caminho em $X$. Nós podemos formar um novo caminho $\langle H, \alpha\rangle: I \rightarrow Y$ definindo $\langle H, \alpha\rangle(t)=H(t)(\alpha(t))$, onde $H(t)(x)=H(x, t)$. 
Teorema 1.2.5 Seja $H: X \times I \rightarrow X$ uma homotopia de $f$ para $g$. Seja $x_{0} \in \operatorname{Fix}(f)$ contido em uma classe de ponto fixo $F$ de $f$ e $x_{1} \in \operatorname{Fix}(g)$ contido em uma classe de ponto fixo $G$ de $g$. Se existe um caminho $\alpha$ em $X$ de $x_{0}$ para $x_{1}$ tal que $\langle H, \alpha\rangle \simeq \alpha \operatorname{rel}\{0,1\}$, então existe uma caminho $\beta$ em $X$ de $x_{0}^{\prime}$ para $x_{1}^{\prime}$ tal que $\langle H, \beta\rangle \simeq \beta \operatorname{rel}\{0,1\}$ para quaisquer $x_{0}^{\prime} \in F$ e $x_{1}^{\prime} \in G$.

Prova: Veja [5], pág 90.

Sejam $f, g: X \rightarrow X$ aplicações sobre um poliedro finito e $H$ uma homotopia de $f$ para $g$. Para uma classe de ponto fixo $F$ de $f$ e uma classe de ponto fixo $G$ de $g$, dizemos que $F$ e $G$ estão H-relacionadas, se existe $x_{0} \in F, x_{1} \in G$ e um caminho $\alpha: I \rightarrow X$ com $\alpha(0)=x_{0}, \alpha(1)=x_{1}$ tal que $\langle H, \alpha\rangle \simeq \alpha \operatorname{rel}\{0,1\}$. Escrevemos $F \mathbf{H} G$ neste caso. O teorema 1.2.5 mostra que a definição é independente da escolha de $x_{0}$ e $x_{1}$.

Teorema 1.2.6 Seja $H$ uma homotopia de $f$ para $g$. Seja $F, F^{\prime}$ classes de ponto fixo de $f$ e $G, G^{\prime}$ classes de ponto fixo de $g$. Se $F \mathbf{H} G$ e $F \mathbf{H} G^{\prime}$ então $G=G^{\prime}$, e se $F \mathrm{H} G$ e $F^{\prime} \mathrm{H} G$ então $F=F^{\prime \prime}$.

Prova: Veja [5], pág 92.

Teorema 1.2.7 Seja $H$ uma homotopia entre $f, g: X \rightarrow X$, onde $X$ é um poliedro finito conexo. Seja $F$ uma classe de ponto fixo de $f$. Se $F$ é $H$ relacionada com alguma classe de ponto fixo $G$ de $g$, então $i(F)=i(G)$; e se $F$ não é $H$-relacionada com qualquer classe de $g$, então $i(F)=0$.

Prova: Veja [5], pág 94.

Os teoremas 1.2.6 e 1.2.7 estabelecem uma correspondência injetiva entre as classes essenciais de $f$ e as classes essenciais de $g$. 
Teorema 1.2.8 (Invariância homotópica do número de Nielsen) Seja X um poliedro finito conexo e $f, g: X \rightarrow X$ aplicações homotópicas; então $N(f)=$ $N(g)$.

Prova: Seja $H$ uma homotopia entre $f$ e $g$. Pelos teoremas 1.2 .6 e 1.2.7 $H$ induz uma correspondência injetiva entre as classes essenciais de $f$ e as classes essenciais de $g$, onde a correspondência é dada pela $H$-relação. Dá $N(f) \leq N(g)$. Considerando agora a homotopia $H^{-1}$ entre $g$ e $f$, pelo mesmo raciocínio obtemos $N(g) \leq N(f)$. Portanto $N(f)=N(g)$.

Lema 1.2.1 Sejam $X$ e $Y$ espaços. Se $f: X \rightarrow Y$ e $g: Y \rightarrow X$ são aplicações, então $f$ e $g$ são homeomorfismos mutuamente inversos entre os conjuntos de pontos fixos $\operatorname{Fix}(g \circ f)(\subseteq X)$ e $\operatorname{Fix}(f \circ g)(\subseteq Y)$.

Lema 1.2.2 Se $f: X \rightarrow Y$ e $g: Y \rightarrow X$ são aplicações entre poliedros finitos e $x_{0}$ e $x_{1}$ são dois pontos fixos de $g \circ f$, então $x_{0}, x_{1}$ pertencem a mesma classe de ponto fixo de $g \circ f$ se, e somente se, $f\left(x_{0}\right)$ e $f\left(x_{1}\right)$ pertence a mesma classe de ponto fixo de $f \circ g$.

Prova: Se existe uma caminho $\alpha$ de $x_{0}$ para $x_{1}$ tal que $g \circ f \circ \alpha \simeq$ $\alpha$ rel $\{0,1\}$, então para o caminho $f \circ \alpha$ de $f\left(x_{0}\right)$ para $f\left(x_{1}\right)$ nós temos $f \circ \alpha \simeq$ $f \circ(g \circ f \circ \alpha) \operatorname{rel}\{0,1\}=(f \circ g) \circ(f \circ \alpha) \operatorname{rel}\{0,1\}$. Reciprocamente se existe um caminho $\beta$ de $f\left(x_{0}\right)$ para $f\left(x_{1}\right)$ tal que $f \circ g \circ \beta \simeq \beta$ rel $\{0,1\}$, temos que $g \circ \beta$ é um caminho de $x_{0}$ para $x_{1}$ e $g \circ(f \circ g \circ \beta)=(g \circ f) \circ(g \circ \beta) \simeq g \circ \beta \operatorname{rel}\{0,1\}$.

Teorema 1.2.9 Se $f: X \rightarrow Y$ e $g: Y \rightarrow X$ são aplicações entre poliedros finitos conexos, então a $f$-imagem de uma classe de ponto fixo de $g \circ f: X \rightarrow X$ é uma classe de ponto fixo de $f \circ g: Y \rightarrow Y$, e assim $f$ estabelece uma correspondência bijetora entre as classes de ponto fixo de 
$g \circ f e$ as classes de ponto fixo de $f \circ g$. Mais ainda, os índices das classes correspondentes são iguais.

Prova: Seja $F$ uma classe de ponto fixo de $g \circ f$. Dos lemas 1.2.1 e 1.2.2, $f(F)$ é uma classe de ponto fixo $F^{\prime}$ de $f \circ g$, e $f: F \rightarrow F^{\prime}$, $g: F^{\prime} \rightarrow F$ são homeomorfismos mutuamente inverso. Resta apenas mostrar que $i(F)=i\left(F^{\prime}\right)$. Sabemos que existe um aberto $U$ de $X$ tal que $F \subseteq U$ e $\bar{U} \cap \operatorname{Fix}(g \circ f)=F$. Como $g\left(F^{\prime}\right)=F$, temos $F^{\prime} \subseteq g^{-1}(U)$. Resta mostrar que $g^{-1}(U) \cap \operatorname{Fix}(f \circ g)=F^{\prime}$. De fato, seja $y \in g^{-1}(U)$ um ponto fixo de $f \circ g$, então $g(y) \in U$ é um ponto fixo de $g \circ f$, isto é; $g(y) \in U \cap \operatorname{Fix}(g \circ f)=F$, e daí $y=f \circ g(y) \in f(F)=F^{\prime}$. Como $i(F)=i(X, g \circ f, U)$ e $i\left(F^{\prime}\right)=$ $i\left(Y, f \circ g, g^{-1}(U)\right)$. Pela comutatividade nós temos $i(F)=i(X, g \circ f, U)=$ $i\left(Y, f \circ g, g^{-1}(U)\right)=i\left(F^{\prime}\right)$.

Teorema 1.2.10 (Comutatividade do número de Nielsen) Se $f: X \rightarrow Y$ e $g: Y \rightarrow X$ são aplicações entre poliedros finitos e conexos, então $f$ estabelece uma correspondência bijetiva entre as classes de ponto fixo essenciais de $g \circ f$ $e$ as classes de ponto fixo essenciais de $f \circ g$, consequentemente

$$
N(g \circ f)=N(f \circ g) \text {. }
$$

\subsection{Teorema de Minimização}

O objetivo central desta seç̧ão é descrever o problema de determinar o número mínimo de pontos fixos e apresentar alguns resultados que serão utilizados no próximo capítulo.

Inicialmente vamos estabelecer uma notação para alguns elementos usuais: 
- se $K$ é um complexo simplicial denotamos por $|K|$ sua realização geométrica, para um simplexo $s \in K,|s|$ será seu interior em $|K|$.

- para $x \in|K|, \sigma(x)$ denota o único simplexo de $K$ tal que $x \in|\sigma(x)|$.

- para $x \in|K|, V(x)$ será a união de todos $|t| \operatorname{com} t \in K$ e $\overline{|t|} \cap \overline{\sigma(x)} \neq \emptyset$.

- $[x, y]$ será o segmento de reta ligando $x$ à $y$.

- para um simplexo $s \in K$, defina $S t(s)$ como a união de todos os simplexos de $K$ que contém $s$, daí

$$
|S t(s)|=\bigcup_{s \subseteq t}|t|
$$

é aberto em $|K|$. St $(s)$ é chamado estrela de $s$.

- seja $A$ um subconjunto de $|K|$ e $\varepsilon>0$, definimos

$$
U(A, \varepsilon)=\{x \in|K| \mid d(x, A)<\varepsilon\}
$$

onde $d$ é uma métrica em $|K| . \bar{U}(A, \varepsilon)$ denotará o fecho de $U(A, \varepsilon)$.

Seja $X$ um poliedro finito conexo. Dada uma aplicação $f: X \rightarrow X$, denotemos por $[f]$ a classe de homotopia de $f$ e \#Fix $(f)$ a cardinalidade de Fix $(f)$. Consideremos o problema de determinar

$$
\mu(f)=\inf \{\# \operatorname{Fix}(g) \mid g \in[f]\}
$$

Em vista da invariância homotópica podemos concluir que $\mu(f) \geq N(f)$, isto é, o número de Nielsen é um limitante inferior para o número de pontos fixos de todas as funções homotópicas a $f$. 
O próximo teorema a ser enunciado nos diz que se o poliedro $X$ satisfazer certas condições, então o número de Nielsen é de fato o melhor limitante inferior.

Um poliedro finito conexo $|K|$ é do tipo $S$ se a dimensão de $K$ é pelo menos três e para cada vértice $v$ de $K$, bordo de $|S t(v)|$ é conexo. Um poliedro $X$ é do tipo $S$ se é homeomorfo a algum $|K|$ do tipo $S$.

Teorema 1.3.1 (Teorema de Minimização) Se X é um poliedro finito conexo do tipo $S$ e $f: X \rightarrow X$ é uma aplicação, então existe uma aplicação $g: X \rightarrow X$ homotopica a $f$ tal que $g$ tem exatamente $N(f)$ pontos fixos.

Prova: Veja [5], pág 140.

A demonstração deste teorema faz uso dos resultados que passaremos a expor. Estes, por sua vez, também nos serão úteis no próximo capítulo.

Seja $K$ um complexo simplicial. Um simplexo $s \in K$ é dito ser maximal se não existe simplexo de $K$ que contém $s$ propriamente.

Teorema 1.3.2 Seja $X$ um poliedro finito conexo de dimensão $\geq 1, e$ $f: X \rightarrow X$ uma aplicação. Dado $\varepsilon>0$, existe uma aplicação $g: X \rightarrow X$ tal que:

1) g tem somente um número finito de pontos fixos;

2) existe uma triangulação $(K, \tau)$ de $X$ tal que cada ponto fixo de $g$ esta em um conjunto $\tau|s|$ para algum simplexo maximal $s \in K$;

3) $d(f, g)<\varepsilon$.

Prova: Veja [5], pág 118.

Concluímos do corolário 1.2.1, que dado $\eta>0$ e $f: X \rightarrow X$ nas condições do teorema acima, existe $g: X \rightarrow X$ tal que $f$ e $g$ são $\eta$-homotópicas e $g$ tem um número finito de pontos fixos que estão em simplexos maximais. 
Teorema 1.3.3 Seja $|K|$ um poliedro finito conexo, $s$ um simplexo maximal de $K, f:|K| \rightarrow|K|$ uma aplicação, $x \in|s|$ um ponto fixo isolado de $f, e$ $U \subseteq|s|$ uma vizinhança de $x$ tal que $\operatorname{Fix}(f) \cap \bar{U}=x$. Se $i(|K|, f, U)=0$ então, dado $\varepsilon>0$, existe uma aplicação $g:|K| \rightarrow|K|$ com as propriedades: 1) $f(y)=g(y)$ para todo $y \in|K|-U$;

2) $d(f, g)<\varepsilon$;

3) $\operatorname{Fix}(g) \cap \bar{U}=\emptyset$.

Prova: Veja [5], pág 123.

Este teorema diz resumidamente que pontos fixos com índice zero podem ser eliminados.

Definiçāo 1.3.1 Para $A \subseteq|K|$, dizemos que uma aplicação $f:|K| \rightarrow|K|$ é próxima em $A$ se $f(x) \in V(x)$ para todo $x \in A$.

Suponha que $f, g:|K| \rightarrow|K|$ são aplicações e que $A \subseteq|K|$ tal que $f(x)=g(x)$ para todo $x \in A$. Se existe uma aplicação $H:|K| \times I \rightarrow|K|$ com as propriedades $H(x, 0)=f(x), H(x, 1)=g(x)$ para todo $x \in|K| \mathrm{e}$ $H(x, t)=f(x)=g(x)$ para todo $x \in A, t \in I$, então dizemos que $f$ e $g$ são homotópicas relativas ao conjunto $A$. Notação: $f \simeq g \operatorname{rel} A$.

Teorema 1.3.4 Dado um poliedro finito conexo $|K|, x \in|K|$ com $\sigma(x)$ maximal, uma aplicação $f:|K| \rightarrow|K|$ e um número real $\eta>0$ tal que $U(x, \eta) \subseteq|\sigma(x)|, \operatorname{Fix}(f) \cap \operatorname{Bd} U(x, \eta)=\emptyset$ e f é próxima em $\bar{U}(x, \eta)$, então existe uma aplicação $g:|K| \rightarrow|K|$ tal que:

1) $g \simeq f \operatorname{rel}|K|-U(x, \eta)$;

2) $\operatorname{Fix}(g) \cap \bar{U}(x, \eta)=x$;

3) $g$ é próxima em $\bar{U}(x, \eta)$. 
Prova: Veja [5], pág 126.

Em outras palavras, se as hipóteses do teorema acima forem satisfeitas e se $f$ eventualmente tiver ponto fixo em $U(x, \eta)$, estes pontos fixos podem ser unidos em um único ponto.

Observaçāo 1.3.1 Na demonstração do teorema 1.3.4 fica claro que $g(y) \in$ $|\sigma(y)| \cup|\sigma(f(y))| \cup|\sigma(y) \cap \sigma(f(y))|$, para todo $y \in|K|$. Este fato será usada na demonstração do lema 2.2.4.

Teorema 1.3.5 Considere um poliedro finito conexo $|K|, s_{1}, s_{2} \in K$ simplexos de dimensão maior do que um tal que $s_{1} \cap s_{2}$ tem dimensão maior do que zero. Se a $\in\left|s_{1}\right|, b \in\left|s_{1} \cap s_{2}\right|$, e se $f:|K| \rightarrow|K|$ é uma aplicação tal que:

1) a é um ponto fixo isolado de $f, i(|K|, f, a) \neq 0$ e $\operatorname{Fix}(f) \cap[a, b]=a$;

2) $f$ é uma aplicação próxima em $[a, b]$.

Então existe $\varepsilon>0$ e uma aplicação $g:|K| \rightarrow|K|$ com as propriedades:

3) $g \simeq f \operatorname{rel}|K|-U([a, b], \varepsilon)$;

4) $\operatorname{Fix}(g) \cap \bar{U}([a, b], \varepsilon)=c$ para algum $c \in U(b, \varepsilon) \cap\left|s_{2}\right|$;

5) g é próxima em $\bar{U}([a, b], \varepsilon)$.

Prova: Veja [5], pág 128.

O teorema 1.3.5 afirma que podemos mover o ponto fixo $a$ ao longo do segmento $[a, b]$ para o ponto fixo $c$ do simplexo $\left|s_{2}\right|$ sem criar novos pontos fixos. 


\section{Capítulo 2}

\section{O Número de Nielsen Relativo}

O objetivo desta secção é introduzir o número de Nielsen relativo e obter algumas consequências imediatas da definição.

\subsection{Definição e Propriedades}

Trabalharemos na categoria de pares de espaços topológicos $(X, A), A \subset X$ e aplicações entre estes pares $f:(X, A) \rightarrow(X, A)$, isto é, função contínua $f: X \rightarrow X \operatorname{com} f(A) \subset A$. As homotopias entre tais aplicações são aplicações contínuas da forma $H:(X \times I, X \times A) \rightarrow(X, A)$. O símbolo $\bar{f}: A \rightarrow A$ representa a restrição de $f$ para $A$. A menos que se fale o contrário $X$ será um poliedro finito conexo e $A$ um subpoliedro. Denotaremos por $d$ a métrica baricêntrica.

Definiçāo 2.1.1 Seja $f:(X, A) \rightarrow(X, A)$ uma aplicação. Uma classe de ponto fixo $F$ de $f: X \rightarrow X$ é uma classe comum de $f$ e $\vec{f}$ se esta contém uma classe de ponto fixo essencial de $\bar{f}: A \rightarrow A$.

Lema 2.1.1 Sejam $f:(X, A) \rightarrow(X, A)$ uma aplicação, $F$ uma classe de ponto fixo de $f: X \rightarrow X$ e $\bar{F}$ uma classe de ponto fixo de $\bar{f}: A \rightarrow A$. Se 
$F \cap \bar{F} \neq \emptyset$, então $\bar{F} \subset F$.

Prova: Dois pontos fixos $x_{0}, x_{1}$ pertencem a $F$ se existe um caminho $\alpha$ em $X$ de $x_{0}$ para $x_{1}$ tal que $f \circ \alpha \simeq \alpha$ rel $\{0,1\}$. Daí se $a_{0} \in F \cap \bar{F}$ e $a_{1} \in \bar{F}$, então existe um caminho $\beta$ em $A$ de $a_{0}$ para $a_{1}$ tal que $\bar{f} \circ \beta \simeq \beta \operatorname{rel}\{0,1\}$ em $A$. Como $A \subset X$ isto implica $a_{1} \in F$.

Definiçāo 2.1.2 Uma classe comum essencial de $f$ e $\bar{f}$ é uma classe de ponto fixo essencial de $f$ que é uma classe comum de $f$ e $\bar{f}$.

Escrevemos $N(f, \bar{f})$ para o número de classes comuns essenciais de $f$ e $\bar{f}$. Note que $N(f, \bar{f})$ é finito, pois $0 \leq N(f, \bar{f}) \leq N(f)$.

Definiçāo 2.1.3 Seja $(X, A)$ um par de espaços. Se $f:(X, A) \rightarrow(X, A)$ é uma aplicação, então o número de Nielsen relativo $N(f ; X, A)$ é definido por

$$
N(f ; X, A)=N(\bar{f})+N(f)-N(f, \bar{f})
$$

Os resultados a seguir são consequências imediatas da definição e de alguns fatos citados na capítulo anterior.

Lema 2.1.2 Seja $(X, A)$ um par de espaços e $f:(X, A) \rightarrow(X, A)$ uma aplicação. Temos que:

1) $S e N(f)=0$, então $N(f ; X, A)=N(\bar{f})$,

2) $S e N(\bar{f})=0$, então $N(f ; X, A)=N(f)$.

Teorema 2.1.1 Toda aplicação $f:(X, A) \rightarrow(X, A)$ tem pelo menos $N(f ; X, A)$ pontos fixos.

Prova: Suponhamos que $\bar{f}: A \rightarrow A$ tenha $m$ classes de pontos fixos essenciais $\bar{F}_{1}, \bar{F}_{2}, \ldots, \bar{F}_{m}$, e $f: X \rightarrow X$ tenha $r$ classes de pontos fixos essenciais $F_{1}, F_{2}, \ldots, F_{n}, F_{n+1}, \ldots, F_{r}$ onde $F_{n+1}, F_{n+2}, \ldots, F_{r}$ são as classes comuns 
de $f$ e $\vec{f}$. Então

$$
N(f ; X, A)=m+r-(r-n)=m+n .
$$

Cada classe de ponto fixo $\bar{F}_{i}$ contém pelo menos um ponto fixo $a_{i}$ de $\bar{f}$, e cada classe de ponto fixo $F_{j}$ contém pelo menos um ponto fixo $x_{j}$ de $f$. Em virtude do lema 2.1.1 $\bar{F}_{i} \cap F_{j}=\emptyset$ para todo $i=1, \ldots, m$ e todo $j=1, \ldots, n$. Portanto o conjunto $\left\{a_{1}, a_{2}, \ldots, a_{m}, x_{1}, x_{2}, \ldots, x_{n}\right\}$ consiste de $m+n$ pontos fixos distintos da aplicação $f:(X, A) \rightarrow(X, A)$.

Teorema 2.1.2 : Se $(X, A)$ é um par de espaços e $f:(X, A) \rightarrow(X, A)$ é uma aplicação, então $N(f ; X, A) \geq N(f)$ e $N(f ; X, A) \geq N(\bar{f})$.

Prova: Como $N(f, \bar{f})$ conta o número de classes de pontos fixos essenciais de $f$ que contém pelo menos uma classe de ponto fixo essencial de $\bar{f}$, temos $N(f, \bar{f}) \leq N(\bar{f})$, daí $N(f ; X, A)=N(f)+[N(\bar{f})-N(f, \bar{f})] \geq N(f)$. Já tinhamos que $N(f, \bar{f}) \leq N(f)$, portanto $N(f ; X, A) \geq N(\bar{f})$.

Teorema 2.1.3 (Invariância homotópica do número de Nielsen relativo) Se as aplicações $f_{0}, f_{1}:(X, A) \rightarrow(X, A)$ são homotópicas, então $N\left(f_{0} ; X, A\right)=$ $N\left(f_{1} ; X, A\right)$.

Prova: Seja $H:(X \times I, A \times I) \rightarrow(X, I)$ uma homotopia entre $f_{0}$ e $f_{1}$. Como já sabemos $N\left(f_{0}\right)=N\left(f_{1}\right)$ e $N\left(\bar{f}_{0}\right)=N\left(\bar{f}_{1}\right)$, é suficiente mostrar que $N(f, \bar{f})$ é um invariante homotópico. Seja $F_{0}$ uma classe comum essencial de $f_{0}$ e $\bar{f}_{0}$. Então $F_{0}$ contém uma classe de ponto fixo essencial $\bar{F}_{0}$ de $\bar{f}_{0}$, que pelo teorema 1.2.7 está $\bar{H}$-relacionada com alguma classe de ponto fixo essencial $\bar{F}_{1}$ de $\bar{f}_{1}$, onde $\bar{H}$ é a restrição de $H$ para $A \times I$. Isto significa que, para todo $a_{0} \in \bar{F}_{0}$ e $a_{1} \in \bar{F}_{1}$, existe um caminho $\alpha$ em $A$ de $a_{0}$ para $x_{1}$ tal que $\langle\bar{H}, \alpha\rangle=\alpha \operatorname{rel}\{0,1\}$ em $A$. Agora seja $F_{1}$ a classe de ponto fixo de $f_{1}$ que contém $a_{1}$. Como $\alpha$ é um caminho em $X$ de $a_{0}$ para $a_{1}$ e 
como $\langle\bar{H}, \alpha\rangle=\langle H, \alpha\rangle$, a classe de ponto fixo $F_{0}$ de $f_{0}$ e $F_{1}$ de $f_{1}$ estão $H$-relacionadas. Segue do teorema 1.2.7 que $F_{1}$ é uma classe de ponto fixo essencial de $f_{1}$, e como $F_{1} \cap \bar{F}_{1} \neq \emptyset$, esta é uma classe comum essencial de $f_{1}$ e $\bar{f}_{1}$. Assim $H$ relaciona classes comuns essenciais de $f_{0}$ e $\bar{f}_{0}$ com classes comuns essenciais de $f_{1}$ e $\bar{f}_{1}$, e portanto temos $N\left(f_{0}, \bar{f}_{0}\right)=N\left(f_{1}, \bar{f}_{1}\right)$.

Corolário 2.1.1 Seja $(X, A)$ um par de espaços e $f:(X, A) \rightarrow(X, A)$ uma aplicação. Se $X$ é simplesmente conexo ou $f$ é homotópica a aplicação identidade id $:(X, A) \rightarrow(X, A)$, então

$$
N(f ; X, A)= \begin{cases}N(f) & \text { se } N(\bar{f})=0 \\ N(\bar{f}) & \text { se } N(\bar{f}) \neq 0\end{cases}
$$

Prova: Temos que considerar somente o caso onde $N(f) \neq 0$ e $N(\bar{f}) \neq 0$. Se $X$ é simplesmente conexo, então $f: X \rightarrow X$ tem uma única classe de ponto fixo essencial $F$, e $\bar{f}: A \rightarrow A$ tem pelo menos uma classe de ponto fixo essencial $\bar{F}$. Mas se $x \in F$ e $a \in \bar{F}$, então $a$ também é ponto fixo de $f: X \rightarrow X$ e esta na mesma classe de ponto fixo de $x$, portanto $N(f, \widehat{f})=1$. Se $f$ é homotópica a identidade, como estamos supondo $N(f) \neq 0$, temos que $N(f)=N(i d)=1$. Pela teorema anterior $N(f, \bar{f})=N(i d, i \bar{d})=1$. Segue que $N(f ; X, A)=N(\bar{f})+1-1=N(\bar{f})$.

Teorema 2.1.4 (Comutatividade do número de Nielsen relativo) Sejam $(X, A)$ $e(Y, B)$ dois pares de espaços. Se $f:(X, A) \rightarrow(Y, B)$ e $g:(Y, B) \rightarrow(X, A)$ são aplicações, então $N(g \circ f, \bar{g} \circ \bar{f})=N(f \circ g, \bar{f} \circ \bar{g})$ e daí

$$
N(g \circ f ; X, A)=N(f \circ g ; Y, B) .
$$

Prova: Seja $F_{0}$ uma classe comum essencial de $g \circ f$ e $\bar{g} \circ \bar{f}$. Então $F_{0}$ contém uma classe de ponto fixo essencial $\bar{F}_{0}$ de $\bar{g} \circ \bar{f}$, pelo teorema 1.2.9, 
$f\left(F_{0}\right)$ é uma classe de ponto fixo essencial de $f \circ g$ e $f\left(\bar{F}_{0}\right)$ é uma classe de ponto fixo essencial de $\bar{f} \circ \bar{g}$. Como $f\left(\bar{F}_{0}\right) \subset f\left(F_{0}\right), f\left(F_{0}\right)$ é uma classe comum essencial de $f \circ g$ e $\bar{f} \circ \bar{g}$. Portanto $f$ estabelece uma correspondência bijetiva entre as classes comuns essenciais de $g \circ f$ e $\bar{g} \circ \bar{f}$ e as classes comuns essenciais de $f \circ g$ e $\bar{f} \circ \bar{g}$. Temos então $N(g \circ f, \bar{g} \circ \bar{f})=N(f \circ g, \bar{f} \circ \bar{g})$. Como já temos $N(f \circ g)=N(g \circ f)$ e $N(\bar{f} \circ \bar{g})=N(\bar{g} \circ \bar{f})$, segue que $N(g \circ f ; X, A)=N(f \circ g ; Y, B)$.

Uma aplicação $h:(X, A) \rightarrow(Y, B)$ é uma equivalência de homotopia quando existe $k:(Y, B) \rightarrow(X, A)$ tal que $k \circ h$ é homotópica à $i d:(X, A) \rightarrow$ $(X, A)$ e $h \circ k$ é homotópica à $i d:(Y, B) \rightarrow(Y, B)$.

Definiçāo 2.1.4 Duas aplicações de pares de espaços $f:(X, A) \rightarrow(X, A)$ e $g:(Y, B) \rightarrow(Y, B)$ são ditas aplicaçōes de mesmo tipo de homotopia se existe uma equivalência de homotopia $h:(X, A) \rightarrow(Y, B)$ tal que as aplicações de pares de espaço $h \circ f, g \circ h:(X, A) \rightarrow(Y, B)$ são homotópicas.

Teorema 2.1.5 Seja $(X, A) e(Y, B)$ dois pares de espaços. Se $f:(X, A) \rightarrow$ $(X, A)$ e $g:(Y, B) \rightarrow(Y, B)$ são aplicações de mesmo tipo de homotopia, então $N(f ; X, A)=N(g ; Y, B)$.

Prova: Usando a mesma notação acima, da invariância homotópica e comutatividade; do número de Nielsen e do número de Nielsen relativo respectivamente, temos que:

$N(f)=N((k \circ h) \circ f)=N(k \circ(h \circ f))=N((h \circ f) \circ k)=N(g)$,

$N(\bar{f})=N((\bar{k} \circ \bar{h}) \circ \bar{f})=N(\bar{k} \circ(\bar{h} \circ \bar{f}))=N((\bar{h} \circ \bar{f}) \circ \bar{k})=N(\bar{g})$,

$N(f, \bar{f})=N((k \circ h) \circ f,(\bar{k} \circ \bar{h}) \circ \bar{f})=N((h \circ f) \circ k,(\bar{h} \circ \bar{f}) \circ \bar{k})=N(g, \bar{g})$,

pois $f$ é homotópica à $(k \circ h) \circ f$ e $(h \circ f) \circ k$ é homotópica à $g$. Asssim temos $N(f ; X, A)=N(g ; Y, B)$. 
Vamos agora para alguns exemplos calcular $N(f ; X, A)$.

Exemplo 2.1.1 Seja $X=B^{n}$, onde $n \geq 2$, a bola fechada $n$-dimensional e $A$ consiste do bordo de $B^{n}$ (a esfera $(n-1)$-dimensional) junto com $k$ pontos do interior de $B^{n}$. Se $i d:(X, A) \rightarrow(X, A)$ é a aplicação identidade, então $\overrightarrow{i d}: A \rightarrow A$ tem $k+1$ classes de ponto fixo, que são os $k$ pontos mais toda a esfera $S^{n-1}$. Todas as classes que contém apenas um ponto são essenciais, pois neste caso temos uma aplicação constante. Resta sabermos se $S^{n-1}$ é essencial. Pelo propriedade de normalização sabemos que o índice

$$
i\left(S^{n-1}\right)=i\left(S^{n-1}, i d, S^{n-1}\right)=L(i d)= \begin{cases}0 & \text { se } n-1 \text { é impar } \\ 2 & \text { se } n-1 \text { é par }\end{cases}
$$

Daí

$$
N(\overline{i d})= \begin{cases}k & \text { se } \mathrm{n} \text { é par } \\ k+1 & \text { se } \mathrm{n} \text { é impar }\end{cases}
$$

e segue do corolário 2.1.1 que

$$
N(i d ; X, A)= \begin{cases}1 & \text { se } k=0 \\ k & \text { se } k \geq 1 \text { e n é par } \\ k+1 & \text { se } k \geq 1 \text { e n é impar }\end{cases}
$$

Suponha que $i: A \rightarrow X$ é a inclusão do subespaço $A$ de $X$. Se existe uma retração $r: X \rightarrow A$ tal que $i \circ r$ é homotópica à identidade, dizemos que $A$ é um retrato por deformaçāo de $X$.

Exemplo 2.1.2 Seja $X=T_{s}^{2}$ o toro sólido em $\mathbb{R}^{3}$ que é obtido pela rotação de um disco de raio 1 e centro $(2,0,0)$ do plano $x z$ em torno do eixo $z$, e seja $A=T^{2}$. Se considerarmos $\mathbb{R}^{3}$ como $\mathbb{C} \times \mathbb{R}$, onde $\mathbb{C}$ é o plano complexo, podemos rotular os pontos de $X$ como $\left(r e^{i \theta}, t\right)$, onde $r e^{i \theta} \in \mathbb{C}$ e $t \in \mathbb{R}$, 
com $1 \leq r \leq 3,0 \leq \theta<2 \pi$ e $-1 \leq t \leq 1$. Seja $f:(X, A) \rightarrow(X, A)$ a aplicação dada por $f\left(r e^{i \theta}, t\right)=\left(r e^{i d \theta},-t\right)$, onde $d \neq 1$ é um inteiro. Como o círculo $S=\left(2 e^{i \theta}, 0\right), 0 \leq \theta<2 \pi$ é um retrato por deformação de $X$, temos que a inclusão $i: S \rightarrow X$ é uma equivalência de homotopia. Portanto a aplicação $f$ e a restrição $\tilde{f}: S \rightarrow S$ de $f$ para $S$ tem o mesmo tipo de homotopia. Pelo teorema 2.1.5 temos $N(f)=N(\widetilde{f})$, e o exemplo 1.2.1 nos $\operatorname{diz}$ que $N(\tilde{f})=|d-1|$. Considere os geradores $[\xi],[\eta]$ de $H_{1}\left(T^{2}\right)=\mathbb{Z} \oplus \mathbb{Z}$, onde $\xi=\left\{\left(2 e^{i \theta}, 1\right) \mid 0 \leq \theta<2 \pi\right\}$ e $\eta=\left\{\left(r e^{i 0}, t\right) \mid(r-2)^{2}+t^{2}=1\right\}$, temos que $\bar{f}_{1 .}[\xi]=d[\xi]$ e $\bar{f}_{1 *}[\eta]=-[\eta]$. Pelo exemplo $1.2 .2 N(\bar{f})=2|d-1|$, que é o valor absoluto do determinante :

$$
\left|\begin{array}{cc}
1-d & 0 \\
0 & 2
\end{array}\right| .
$$

Como a equação $e^{i \theta}=e^{d i \theta}$ tem $|d-1|$ soluções distintas, os pontos fixos de $f$ consistem de $|d-1|$ segmentos de reta, pois eles só podem ocorrer em $t=0$. Cada segmento de reta forma uma classe de ponto fixo essencial de $f \mathrm{e}$ contém duas classes de ponto fixo essenciais de $\bar{f}$ sobre seu bordo. Portanto $N(f, \bar{f})=N(f)$ e $N(f ; X, A)=N(\bar{f})=2|d-1|$.

Exemplo 2.1.3 Seja $X$ um disco com dois buracos, $A$ seu bordo e $f$ a reflexão em torno do eixo $l$. Considerando $X$ como a união $U \cup V$, onde $U$ é o lado direito de $l$ e $V$ o lado esquerdo (ver figura 2.1); construímos a sequência de Mayer Vietoris. Como $U$ e $V$ tem o mesmo tipo de homotopia que $S^{1}$ e $U \cap V$ é contrátil, temos que: $H_{0}(X)=\mathbb{Z}, H_{1}(X)=\mathbb{Z} \oplus \mathbb{Z}$ e $H_{q}(X)=0$ para todo $q \neq 0,1$. O fato de $f$ ter apenas a classe de ponto fixo $U \cap V$, nos diz que seu índice é igual ao número de Lefschetz, $L(f)$, de $f$. Sejam $[\gamma]$ e $[\zeta]$ os geradores de $H_{1}(X)=\mathbb{Z} \oplus \mathbb{Z}$, onde $\gamma$ e $\zeta$ são indicados na figura (2.1). Temos então $f_{1 .}[\gamma]=-[\zeta], f_{1}[\zeta]=-[\gamma]$ e a matriz de $f_{1}$. nesta 
base é:

$$
\left|\begin{array}{cc}
0 & -1 \\
-1 & 0
\end{array}\right| \text {. }
$$

Portanto $L(f)=1$ e consequentemente $N(f)=1$. Claramente $N(\bar{f})=2 \mathrm{e}$ $N(f, \bar{f})=1$, pois cada extremo de $U \cap V$ constitui uma classe de ponto fixo de $\bar{f}$ e seus índices valem 1 , pelo exemplo 1.1. Assim $N(f ; X, A)=2$.
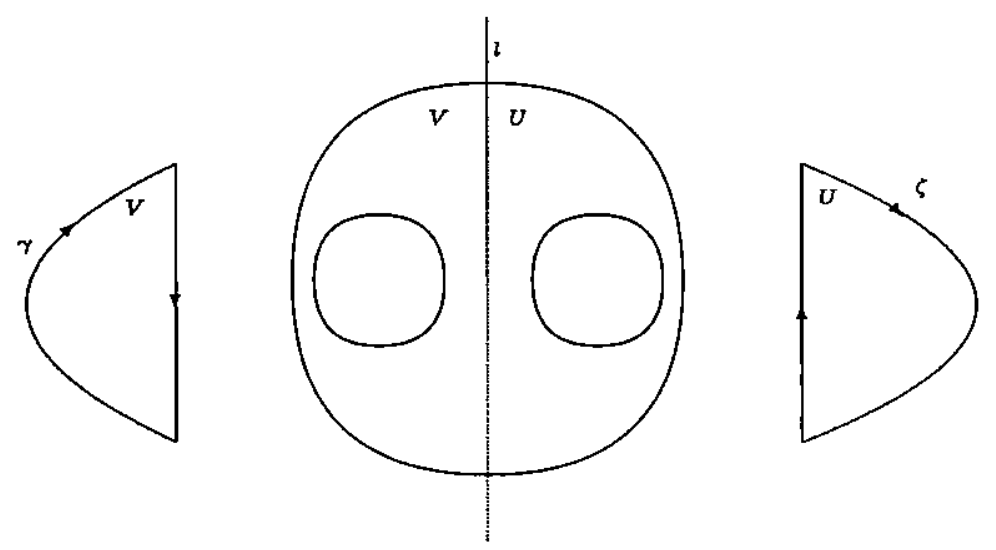

Figura 2.1:

\subsection{Teorema de Minimização Relativo}

Definiçāo 2.2.1 Seja $X$ um espaço topológico e $A$ um subespaço. Uma homotopia $H: A \times I \rightarrow X$ é especial se as aplicações $\phi_{t}(x)=H(x, t)$ tem os mesmos pontos fixos para todo $t \in I$. Duas aplicações $f_{0}, f_{1}: A \rightarrow X$ tendo os mesmos pontos fixos são especialmente homotópicas se podem ser ligadas por uma homotopia especial.

Lema 2.2.1 Seja $X$ um poliedro $e A \subset X$ um subpoliedro. Então existe uma retração $r: X \times I \rightarrow(X \times 0) \cup(A \times I)$. 
Prova: Veja [7], pág 117.

Teorema 2.2.1 Considere $X$ um poliedro e $A \subset X$ um subpoliedro. Seja $f_{0}: X \rightarrow X$ uma auto aplicação, $H: A \times I \rightarrow X$ uma homotopia especial tal que $H(x, 0)=f_{0}(x)$. Então $H$ pode ser estendida para uma homotopia especial $G: X \times I \rightarrow X$ tal que $G(x, 0)=f_{0}(x)$.

Prova: Defina uma aplicação $\phi$ do subespaço $(X \times 0) \cup(A \times I) \subset X \times I$ em $X$ por

$$
\phi(x, t)= \begin{cases}f_{0}(x) & \text { se } t=0 \\ H(x, t) & \text { se } x \in A .\end{cases}
$$

De acordo com o lema 2.2.1, existe uma retração $r: X \times I \rightarrow(X \times 0) \cup$ $(A \times I)$. Coloque $\Phi=\phi \circ r: X \times I \rightarrow X$. O conjunto $C=\{x \mid x \in \Phi(x \times I)\}$ é fechado em $X,\left(\right.$ pois caso $x_{0} \notin C$, isto é, $x_{0} \notin \Phi\left(x_{0} \times I\right)$; como $\Phi\left(x_{0} \times I\right)$ é compacto, temos $\varepsilon=d\left(x_{0}, \Phi\left(x_{0} \times I\right)>0\right.$ e consequentemente $U\left(x_{0}, \varepsilon / 4\right)$ é disjunto de $B=\bigcup_{x \in \Phi\left(x_{0} \times I\right)} U(x, \varepsilon / 4) \supset \Phi\left(x_{0} \times I\right)$, por continuidade $\Phi^{-1}(B)$ é aberto contendo $x_{0} \times I$, mas $I$ é compacto e portanto existe aberto $V \ni x_{0}$ de $X$ tal que $V \times I \subset \Phi^{-1}(B)$. Portanto $\left.V \cap U\left(x_{0}, \varepsilon / 4\right) \subset X-C\right)$. Note que $C$ é a união de todos os pontos fixos das funções $\Phi_{t}(x)=\Phi(x, t) \operatorname{com} t$ variando em $I$. Defina $G: X \times I \rightarrow X$ por

$$
G(x, t)= \begin{cases}\Phi(x, 0)=f_{0}(x) & \text { se } d(x, A) \geq d(x, C) \\ \Phi(x, t-t d(x, A) / d(x, C)) & \text { se } d(x, A) \leq d(x, C)>0 .\end{cases}
$$

$G$ está bem definida. Se $d(x, A)=d(x, C)=0$, como $A$ e $C$ são fechados $x \in A \cap C$, isto é, $x$ é um ponto fixo de $f_{0}$ em $A$. Seja $V \ni x$ um aberto de $X, \Phi^{-1}(V)$ é aberto de $X \times I$ que contém $x \times I$, pois $\left.\Phi\right|_{A \times I}=H$. O fato de $I$ ser compacto implica a existência de um aberto $W \ni x$ em $X$ tal que $W \times I \subset \Phi^{-1}(V)$, daí $G(W \times I) \subset V$. Isto mostra que $G$ é contínua em 
todo ponto $(x, t)$ com $d(x, A)=d(x, C)=0$. A continuidade de $G$ em outros pontos é óbvia. Assim $G$ é uma homotopia e uma extensão de $H$ para $X \times I$. Note que $G$ é especial, pois os pontos fixos de $g_{t}(x)=G(x, t)$ devem estar em $C$ e $G(x, t)=f_{0}(x)$ para todo $t \in I$ e $x \in C$.

Definiçāo 2.2.2 Seja $i: A \rightarrow X$ a inclusão. O subespaço $A$ é um retrato por deformaçāo forte de $X$ se existe uma retração $r: X \rightarrow A$ tal que $i \circ r \simeq i d \operatorname{rel} A$.

Lema 2.2.2 Se $X$ é um poliedro e $A \subset X$ um subpoliedro, então $A$ é um retrato por deformação forte de alguma vizinhança de $A$ em $X$.

Prova: Veja [7], pág 124.

Definiçāo 2.2.3 Seja $(X, A)$ um par de espaços e $Y$ um espaço . $(X, A)$ é dito ter propriedade de extensāo de homotopia com respeito a $Y$ se, dado aplicações $g: X \rightarrow Y$ e $G: A \times I \rightarrow Y$ tal que $g(x)=G(x, 0)$ para $x \in A$, existe uma aplicação $F: X \times I \rightarrow Y$ tal que $F(x, 0)=g(x)$ para $x \in X$ e F $\left.\right|_{A \times I}=G$.

Lema 2.2.3 Se $X$ é um poliedro e $A \subset X$ um subpoliedro, então $(X, A)$ tem a propriedade de extensão de homotopia com respeito a qualquer espaço.

Prova: Veja [7], pág 118.

Definiçāo 2.2.4 Um poliedro finito $X$ é dito ser um espaço de Nielsen se toda aplicação $f: X \rightarrow X$ é homotópica a alguma aplicação $g: X \rightarrow X$ que tem $N(f)$ pontos fixos, e estes pontos fixos podem localizar-se em qualquer ponto de $X$.

Seja $Y$ espaço topológico e $X \subset Y$ um subconjunto; escrevemos $\bar{X}$, int $X$, Bd $X$ para o fecho, interior e bordo de $X$ em $Y$. 
Teorema 2.2.2 Seja $X$ um poliedro finito conexo, $A \neq X$ um subpoliedro onde cada componente conexa é um espaço de Nielsen. Então toda aplicação $f:(X, A) \rightarrow(X, A)$ é homotópica a alguma aplicação $g:(X, A) \rightarrow(X, A)$ satisfazendo:

1) $\bar{g} \operatorname{tem} N(\bar{f})$ pontos fixos no bordo de $A$,

2) $g$ tem um número finito de pontos fixos,

3) todos os pontos fixos de $g$ em $X-A$ estão em simplexos maximais.

Prova: A demonstração será dada em dois passos.

Passso 1: Mostremos que $f:(X, A) \rightarrow(X, A)$ é homotópica a uma aplicação, $h:(X, A) \rightarrow(X, A)$ que tem as seguintes propriedades:

(a) $\bar{h}$ tem $N(\bar{f})$ pontos fixos sobre o bordo de $A$.

(b) existe um poliedro finito $B$ em $X$ de tal forma que $A \subset X-B$ e $h$ não tem pontos fixos sobre $\overline{(X-B)}-A$.

Para construir $h$, usamos o fato que cada componente conexa de $A$ é um espaço de Nielsen para deformar $\bar{f}: A \rightarrow A$ para uma auto aplicação de $A$ que tem $N(\bar{f})$ pontos fixos sobre o bordo de $A$. Seja $\bar{h}: A \rightarrow A$ a aplicação resultante e $\bar{F}: A \times I \rightarrow A$ uma homotopia de $\bar{f}$ para $\bar{h}$. Pelo lema 2.2 .2 o subpoliedro $A$ é um retrato por deformação forte de alguma vizinhança $V$ de $A$ em $X$. Usando uma cobertura estrela (coleção formada pelas estrelas de cada vértice em $A$ ) de $A$ com respeito a alguma subdivisão da triangulação de $X$, podemos achar um poliedro finito $A_{1} \operatorname{com} A \subset \operatorname{int} A_{1} \subset V$ e tal que $B=X-\operatorname{int} A_{1}$ é um subpoliedro em $X$. Seja $R: A_{1} \times I \rightarrow V$ a restrição para $A_{1}$ do retrato por deformação forte de $V$ sobre $A$ e seja $r: A_{1} \rightarrow A$ a retração dada por $r(x)=R(x, 1)$. Então podemos definir uma homotopia $F_{1}: A_{1} \times I \rightarrow X$ por

$$
F_{1}(x, t)= \begin{cases}f \circ R(x, 2 t) & \text { se } 0 \leq t \leq 1 / 2 \\ \bar{F}(r(x), 2 t-1) & \text { se } 1 / 2 \leq t \leq 1\end{cases}
$$


e usamos a propriedade de extensão de homotopia lema 2.2.3 para extender $F_{1}$ para uma homotopia $F:(X \times I, A \times I) \rightarrow(X, A)$ de $f$. Se definirmos $h:(X, A) \rightarrow(X, A)$ por $h(x)=F(x, 1)$, então $h$ não tem pontos fixos sobre $\overline{(X-B)}-A \subset A_{1}-A$, pois $h\left(A_{1}-A\right) \subset A$.

Passo 2: Agora mostramos que $h:(X, A) \rightarrow(X, A)$ é homotópica a uma aplicação $g:(X, A) \rightarrow(X, A)$ que satisfaz as condições do teorema.

Seja $U=X-B$. Como $h$ não tem pontos fixos sobre $\bar{U}-A$, existe um $\delta>0$ tal que $d(x, h(x))>\delta$ para todo $x$ no bordo de $U$. Com a ajuda do teorema 1.3.2 e corolário 1.2.1 deformamos a restrição $h_{B}: B \rightarrow X$ de $h$ para uma aplicação $g_{B}: B \rightarrow X$ que tem um número finito de pontos fixos contidos em simplexos maximais e que é $\delta$-homotópica à $h_{B}$. Seja $G_{B}: B \times I \rightarrow X$ uma tal $\delta$-homotopia de $h_{B}$ para $g_{B}$. Então $G_{B}(x, t) \neq x$ para todo $x$ pertencente ao bordo de $U$, pois $\operatorname{diam}\left(G_{B}(x \times I)\right)<\delta$ para todo $x \in B$. Se $G^{\prime}:((\bar{U} \times 0) \cup(\operatorname{Bd} U \times I) \cup(A \times I), A \times I) \rightarrow(X, A)$ é dada por

$$
G^{\prime}(x, t)= \begin{cases}h(x) & \text { se }(x, t) \in(\bar{U} \times 0) \cup(A \times I), \\ G_{B}(x, t) & \text { se }(x, t) \in(\operatorname{Bd} U) \times I\end{cases}
$$

então a restrição de $G^{\prime}$ para $(\operatorname{Bd} U \cup A) \times I$ é uma homotopia especial e daí extende-se para uma homotopia especial $G_{U}:(\bar{U} \times I, A \times I) \rightarrow(X, A)$. Definimos uma homotopia $G:(X \times I, A \times I) \rightarrow(X, A)$ por

$$
G(x, t)= \begin{cases}G_{U}(x, t) & \text { se }(x, t) \in \bar{U} \times I \\ G_{B}(x, t) & \text { se }(x, t) \in B \times I\end{cases}
$$

e a aplicação $g:(X, A) \rightarrow(X, A)$ por $g(x)=G(x, 1)$. Note que o fato de $G_{U}$ ser especial, implica que $\bar{g}=\left.g\right|_{A}$ tem os mesmos pontos fixos de $\bar{h}$ e $\left.g\right|_{\bar{U}-A}$ não tem pontos fixos, por isto $\bar{g}$ tem $N(\bar{f})$ pontos fixos que estão no bordo de $A$ e tem apenas um número finito de pontos fixos. Portanto $g$ é a função procurada. 
Definiçāo 2.2.5 Um subespaço $A$ de um espaço $X$ pode ser "by-passed" se todo caminho em $X$ com pontos finais em $X-A$ é homotópico a um caminho em $X-A$.

Escrevemos $i_{*}: \pi_{1}(X-A) \rightarrow \pi_{1}(X)$ para o homomorfismo nos grupos fundamentais induzido pela inclusão.

Teorema 2.2.3 Seja $(X, A)$ um par de espaços e $X$ conexo por caminhos. Então $A$ pode ser by-passed em $X$ se e somente se $X-A$ é conexo por caminhos e $i_{*}: \pi_{1}(X-A) \rightarrow \pi_{1}(X)$ é sobrejetiva.

Prova: Suponhamos $A$ pode ser by-passed. Dados dois pontos $a, b \in$ $X-A$, existe um caminho em $X$ de $a$ para $b$ homotópico à um caminho contido em $X-A$, segue que $X-A$ é conexo por caminhos.

Consideremos um ponto $y \in X-A$ como ponto base. Se $\alpha$ é um caminho em $X$ com ponto inicial e final $y$, como $A$ é can by passed existe um caminho $\beta$ em $X-A$ que começa e termina em $y$ homotópico à $\alpha$, daí $i_{*}(\langle\beta\rangle)=\langle\alpha\rangle$ e $i_{*}$ é sobrejetiva.

Reciprocamente, seja $\lambda$ um caminho em $X$ de $a$ para $b$ onde $a, b \in X-A$, como $X-A$ é conexo por caminhos existe um caminho $\alpha$ em $X-A$ de $b$ para $a$. O caminho $\lambda \alpha$ começa e termina em $a$, o fato de $i_{*}$ ser sobrejetiva implica a existência de um caminho $\beta \operatorname{em} X-A$ homotópico à $\lambda \alpha$, dá $\beta \alpha^{-1}$ é um caminho em $X-A$ de $a$ para $b$ homotópico à $\lambda$.

Definiçäo 2.2.6 Um caminho $P L$ em $|K|$ é um caminho $p: I \rightarrow|K| q u e$, para alguma subdivisão $L$ de $I$, aplica cada simplexo de $L$ linearmente em um simplexo de $K$. A imagem de cada vértice de $L$ é chamado um canto de p. Um caminho PL é normal se:

1) não passa por qualquer vértice de $K$, 
2) não tem múltiplas auto interseções e não tem auto interseções em seus cantos,

3) $p(s)$ está em simplexos maximais de $K$ a menos de um número finito de valores de $s \in I$, e $p(s)$ vai de um simplexo maximal a outro quando s cruza qualquer destes valores.

Um arco PL $q: I \rightarrow|K|$ é um caminho PL com pontos finais diferentes e sem auto interseções.

Se $A$ é um subpoliedro de $X=|K|$, dizemos que $Q=p(I)$ é um arco PL-normal em $(|K|, A)$ se $Q$ é um arco PL-normal em $|K|-A$ ou se $Q \cap A=$ $\{q(1)\}$ e $Q$ é um arco PL-normal em $|K|$ à parte o fato de que $q(1)$ pode ser um ponto arbitrário do bordo de $A$.

Definiçāo 2.2.7 Um ponto $x$ de um espaço topológico $X$ é ponto de corte local se existe um conjunto aberto conexo $U \ni x$ tal que $U-x$ não é conexo.

Um poliedro finito conexo $X$ não tem ponto de corte local se para qualquer triangulação $K$ de $X$, todo simplexo maximal de $K$ é de dimensão maior ou igual à 2 e para todo vértice $v$ de $K$ o bordo de $|S t(v)|$ é conexo.

Lema 2.2.4 Seja $(X, A)=(|K|,|L|)$ um par de poliedros finito, onde $X$ é conexo e $X-A$ não tem ponto de corte local e não é Q-variedade. Sejam $x_{0}$ $e x_{1}$ dois pontos fixos isolados de uma aplicação $f:(X, A) \rightarrow(X, A)$, e seja $Q$ um arco $P L$-normal em $(|K|, A)$ de $x_{0}$ para $x_{1}$, com $\operatorname{Fix}(f) \cap Q=\left\{x_{0}, x_{1}\right\}$. Suponha que $x_{0}$ está em um simplexo maximal de $|K|-A$, e que $x_{1}$ está em um simplexo maximal de $|K|-A$ ou sobre o bordo de $A$. Então existe um $\varepsilon>0$ tal que se $\left.f\right|_{Q}$ é especialmente homotópica a uma aplicação $g: Q \rightarrow X$ com $d(x, g(x))<\varepsilon$ para todo $x \in Q$, então $f$ é homotópica a uma aplicação $f^{\prime}:(X, A) \rightarrow(X, A) \operatorname{com} \operatorname{Fix}\left(f^{\prime}\right)=\operatorname{Fix}(f)-\left\{x_{0}\right\}$. 
Prova: Seja $Q=q(I)$, onde $q: I \rightarrow|K|$. Denote por $|s| \subset|K|-$ $A$ o simplexo maximal tal que $x_{1} \in \overline{|s|}$. Tome um ponto $x_{2} \in Q$ de tal forma que $\left[x_{2}, x_{1}\right) \subset|s|$. Existe um número $\eta>0$ tal que $U\left(\left[x_{2}, x_{1}\right], \eta\right) \subseteq$ $\left|S t\left(\sigma\left(x_{1}\right)\right)\right|$. Considere $\delta>0$ o número de Lebesgue da cobertura de $|K|$ formada pela coleção das estrelas dos vértices de $K$. Se $\varepsilon=\min \{\delta, \eta\}$, temos que $d(x, g(x))<\varepsilon$ para todo $x \in Q$, implica que $g: Q \rightarrow X$ é uma aplicação próxima e $g(x) \in\left|S t\left(\sigma\left(x_{1}\right)\right)\right|$ quando $x \in\left[x_{2}, x_{1}\right]$.

Por hipótese existe uma homotopia especial $G_{Q}: Q \times I \rightarrow X$ de $\left.f\right|_{Q}$ para $g: Q \rightarrow X$. Estendemos $G_{Q}$ para uma homotopia especial $G:\{(Q \cup A) \times I, A \times I\} \rightarrow(X, A)$ da forma

$$
G(x, t)= \begin{cases}G_{Q}(x, t) & \text { se }(x, t) \in Q \times I \\ \bar{f}(x) & \text { se }(x, t) \in A \times I\end{cases}
$$

e usando teorema 2.2.1 com $A \cup Q$ ao invés de $A$ estendemos $G$ para uma homotopia especial $H:(X \times I, A \times I) \rightarrow(X, A)$ que começa em $H(x, 0)=$ $f(x)$. Se $f^{\prime \prime}:(X, A) \rightarrow(X, A)$ é dado por $f^{\prime \prime}(x)=H(x, 1)$, então $f^{\prime \prime}(x)=$ $g(x)$ para $x \in Q$.

Como $g: Q \rightarrow X$ é uma aplicação próxima, podemos usar sucessivamente os teoremas 1.3 .4 e 1.3.5 para mover o ponto fixo $x_{0}$ de $f^{\prime \prime}$ ao longo de $Q$ para o ponto $x_{2}$ de tal maneira que a restrição para $Q$ da nova aplicação permanece próxima. Se $Q \subset X-A$, pelo teorema 1.3.4 podemos unir $x_{2}$ com $x_{1}$ e obter uma aplicação $f^{\prime}:(X, A) \rightarrow(X, A)$ homotópica a $f$ com $\operatorname{Fix}\left(f^{\prime}\right)=\operatorname{Fix}(f)-\left\{x_{0}\right\}$.

Temos ainda que unir os pontos $x_{2}$ e $x_{1}$ no caso $Q \cap A=\left\{x_{1}\right\}$. Seja $f_{1}:(X, A) \rightarrow(X, A)$ a aplicação obtida de $f^{\prime \prime}$ que tem $x_{2} \in|s|$ e $x_{1} \in \overline{|s|} \cap A$ como pontos fixos isolados. Note que, da observação 1.3.1 e do modo como $\varepsilon$ foi obtido, $f_{1}(x) \in\left|S t\left(\sigma\left(x_{1}\right)\right)\right|$ quando $x \in\left[x_{2}, x_{1}\right]$. Como $\operatorname{Fix}\left(f_{1}\right) \cap\left[x_{2}, x_{1}\right]=$ $\left\{x_{2}, x_{1}\right\}$, existe uma vizinhança aberta $U$ de $\left[x_{2}, x_{1}\right)$ em $|s| \operatorname{com} \operatorname{Bd} U \cap A=$ 
$\left\{x_{1}\right\}, \operatorname{Fix}\left(f_{1}\right) \cap \mathrm{Bd} U=\emptyset, \bar{U}$ convexo e $f_{1}(\bar{U}) \subseteq\left|S t\left(\sigma\left(x_{1}\right)\right)\right|$ (ver figura 2.2). Os pontos de $\bar{U}-\left\{x_{1}\right\}$ podem ser rótulados como $x=\left(b_{x}, t_{x}\right)$, onde $b_{x} \in \operatorname{Bd} U$, $0<t_{x} \leq 1$ e $x=t_{x} b_{x}+\left(1-t_{x}\right) x_{1}$.

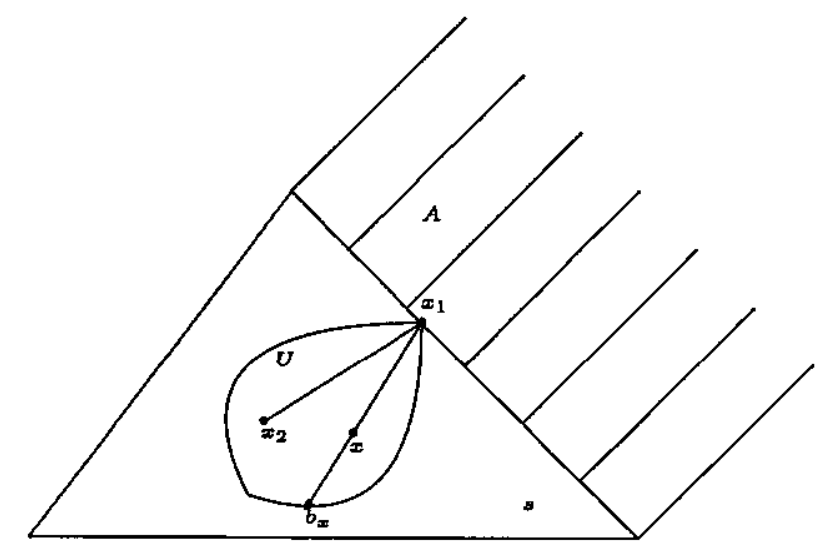

Figura 2.2:

Seja $f^{\prime}:(X, A) \rightarrow(X, A)$ definida por

$$
f^{\prime}(x)= \begin{cases}x_{1} & \text { se } x=x_{1} \\ t_{x} f_{1}\left(b_{x}\right)+\left(1-t_{x}\right) x_{1} & \text { se } x=\left(b_{x}, t_{x}\right) \in \bar{U}-\left\{x_{1}\right\} \\ f_{1}(x) & \text { se } x \in X-\bar{U}\end{cases}
$$

A aplicação $f^{\prime}$ tem somente $x_{1}$ como ponto fixo sobre $\bar{U}$.

Considere a aplicação $H: I \times I \rightarrow X$ definida por

$$
H(x, s)= \begin{cases}f_{1}(x) & \text { se } x \in X-\bar{U} \\ x_{1} & \text { se } x=x_{1} \\ \left(1-s+s t_{x}\right) f_{1}\left(s b_{x}+(1-s) x\right)+s\left(1-t_{x}\right) x_{1} & \text { se } x \in \bar{U}-x_{1}\end{cases}
$$

$H$ é uma homotopia entre $f_{1}$ e $f^{\prime}$. Portanto $f^{\prime}$ é homotópica à $f$ e $\operatorname{Fix}\left(f^{\prime}\right)=\operatorname{Fix}(f)-\left\{x_{0}\right\}$. 
Definiçāo 2.2.8 Seja $q: I \rightarrow|K|$ um arco $P L$-normal e $Q=q(I)$. Dizemos que um caminho $p: I \rightarrow|K|$ é especial com respeito a $q$, se $p(0)=q(0)$, $p(1)=q(1)$ e $p(s) \neq q(s)$ para $0<s<1$. Dois caminhos $p_{0}, p_{1}: I \rightarrow|K|$ são especialmente homotópicos se eles podem ser ligados por uma homotopia $H: I \times I \rightarrow|K|$ onde para cada $t, H_{t}(s)=H(s, t)$ é especial.

Lema 2.2.5 Se os dois pontos finais de $q$ são os únicos pontos fixos sobre $Q$ de $f:|K| \rightarrow|K|$, então o caminho $p: I \rightarrow|K|$ é especialmente homotópico $\grave{a} f \circ q$ se, e somente se, a aplicação $\Phi=p \circ q^{-1}: Q \rightarrow|K|$ é especialmente homotópica a $\left.f\right|_{Q}: Q \rightarrow|K|$ (no sentido da definição 2.2.1).

Prova: Seja $H: I \times I \rightarrow|K|$ a homotopia que liga $p$ à $f \circ q$, onde cada $H_{t}(s)=H(s, t)$ é especial com respeito a $q$. Então $G: Q \times I \rightarrow|K|$ definida por $G(x, t)=H\left(q^{-1}(x), t\right)$ satisfaz:

$$
\begin{aligned}
& G(x, 0)=H\left(q^{-1}(x), 0\right)=p \circ q^{-1}(x) \mathrm{e} \\
& G(x, 1)=H\left(q^{-1}(x), 1\right)=f \circ q \circ q^{-1}(x)=f(x) .
\end{aligned}
$$

Note que $G_{t}(x)=H_{t}\left(q^{-1}(x)\right)$ só tem os pontos finais de $Q$ como pontos fixos. Portanto $G$ é uma homotopia especial entre $p \circ q^{-1}$ e $f$.

Reciprocamente se $G: Q \times I \rightarrow|K|$ for uma homotopia especial entre $p \circ q^{-1}$ e $\left.f\right|_{Q}$. Definimos $H: I \times I \rightarrow|K|$ por $H(s, t)=G(q(s), t)$ temos:

$$
\begin{aligned}
& H(s, 0)=G(q(s), 0)=p \circ q^{-1} \circ q(s)=p(s) \\
& H(s, 1)=G(q(s), 1)=f \circ q(s) \\
& H(0, t)=G(q(0), t)=q(0) \\
& H(1, t)=G(q(1), t)=q(1)
\end{aligned}
$$

e cada $H_{t}(s)=H(s, t)$ é especial com respeito a $q$.

Lema 2.2.6 Seja $|K|$ um poliedro finito conexo sem ponto de corte local. Considere $q: I \rightarrow|K|$ um arco $P L$-normal que satisfaz as seguintes condições: 
( $\alpha$ ) Existe um simplexo $\tau$, sendo um subconjunto aberto de $|K|$, e números $0<s_{1}<s_{3}<s_{2}<1$, tal que $q(s) \in \mathrm{Bd} \tau$ se $s=s_{1}$ ou $s_{2}, q\left(s_{3}\right) \in \tau$ e qé linear sobre $\left[s_{1}, s_{3}\right]$ e $\left[s_{3}, s_{2}\right]$.

( $\beta$ ) $q\left(s_{1}\right)$ e $q\left(s_{2}\right)$ estão em um simplexo $\sigma_{1}$ de dimensão 1 que é face comum de pelo menos dois simplexos maximais e de pelo menos três 2-simplexos de $K$.

Se dois caminhos especiais com respeito à $q, p_{0}, p_{1}: I \rightarrow|K|$ são homotópicos, então eles são especialmente homotópicos.

Prova: Veja apêndice, lema A.6.

Teorema 2.2.4 (Teorema de Minimização Relativo) Seja $X$ um poliedro finito conexo sem pontos de corte local e $A$ um subpoliedro, tal que:

1) $X-A$ não é uma 2-variedade,

2) toda componente conexa de A é um espaço de Nielsen,

3) A pode ser by-passed.

Então qualquer aplicação $f:(X, A) \rightarrow(X, A)$ é homotópica a uma aplicação $g:(X, A) \rightarrow(X, A)$ com $N(f ; X, A)$ pontos fixos.

Prova: Seja $(K, L)$ uma triangulação de $(X, A)$. Devido ao teorema 2.2.2 podemos supor que $\bar{f}$ tem $N(\bar{f})$ pontos fixos que estão sobre o bordo de $A$ e que $f$ tem apenas um número finito de pontos fixos sobre $X-A$ e que todos os pontos fixos de $f$ sobre $X-A$ estão em simplexos maximais de $|K|$. Nós agora uniremos pontos fixos pertencentes a uma classe de ponto fixo $F$ de $f: X \rightarrow X$. Para isto é suficiente mostrar:

$\left.{ }^{*}\right)$ Suponha que $F$ é uma classe comum de $f$ e $\bar{f}$ e $x_{0} \in F \cap(X-A)$ ou que $F$ não é uma classe comum de $f$ e $\bar{f}$ (isto é, $F \cap A=\emptyset$ ) e $x_{0}, x_{1} \in F$. Então $f$ é homotópica a uma aplicação $f^{\prime}:(X, A) \rightarrow(X, A) \operatorname{com} \operatorname{Fix}\left(f^{\prime}\right)=$ 
$\operatorname{Fix}(f)-\left\{x_{0}\right\}$

Repetindo $\left(^{*}\right)$ várias vezes e eliminando as classes de ponto fixo não comuns que consiste de pontos fixos de índice zero, através do teorema 1.3.3, chegamos a uma aplicação $g:(X, A) \rightarrow(X, A) \operatorname{com} N(f ; X, A)$ pontos fixos.

Demonstremos $\left({ }^{*}\right)$ : Seja $F$ uma classe de ponto fixo de $f: X \rightarrow X$ e $x_{0} \in F \cap(X-A)$. Se $F$ é uma classe comum de $f$ e $\bar{f}$, tome $x_{1} \in F \cap B d A$, e se $F$ não é uma classe comum de $f$ e $\bar{f}$, tome $x_{1} \in F-\left\{x_{0}\right\}$. Existe um caminho $q: I \rightarrow X$ de $x_{0}$ para $x_{1}$ que é homotópico à $f \circ q, \operatorname{Fix}(f) \cap Q=\left\{x_{0}, x_{1}\right\}$, tem a propriedade que $Q=q(I)$ é um arco PL-normal em $(|K|, A) \operatorname{com} Q \cap A=\emptyset$ se $x_{1} \in X-A$ e $Q \cap A=\left\{x_{1}\right\}$ se $x_{1} \in \operatorname{Bd} A$ e satisfaz as condições $(\alpha)$ e $(\beta)$ do lema 2.2.6 (veja apêndice, lemas A.1 e A.2, observando que o fato de $X$ não ter ponto de corte local implica que $X-A$ não tem ponto de corte local).

Escolhemos $\varepsilon>0$ de tal forma que o lema 2.2.4 pode ser aplicado. Como $q$ é uniformemente contínua, existe $\delta=\delta(\varepsilon)>0$ tal que se $|h| \leq \delta$, implica $d(q(s+h), q(s))<\varepsilon$, para todo $s \in I$. Considerando este delta, defina $p_{\varepsilon}$ : $I \rightarrow X$ por $p_{\varepsilon}(s)=q(s+\delta \sin (s \pi))$, portanto $d\left(p_{\varepsilon}(s), q(s)\right)<\varepsilon$ para todo $s \in$ I. A aplicação $H: I \times I \rightarrow X$ definida por $H(s, t)=q(s+t \delta \sin (s \pi))$ é uma homotopia ligando $q$ à $p_{\varepsilon}$. O fato de $\operatorname{Fix}(f) \cap Q=\left\{x_{0}, x_{1}\right\}$, implica que $f \circ q$ é um caminho especial com respeito a $Q$, daí $p_{\varepsilon}$ e $f \circ q$ são caminhos especiais e são homotópicos. Segue do lema 2.2.6 que $p_{\varepsilon}$ e $f \circ q$ são especialmente homotópicos. Portanto as aplicações $\left.f\right|_{Q}: Q \rightarrow X$ e $p_{\varepsilon} \circ q^{-1}: Q \rightarrow X$ são especialmente homotópicas e $d\left(x, p_{\varepsilon} \circ q^{-1}(x)\right)=d\left(q(s), p_{\varepsilon}(s)\right)<\varepsilon$ para todo $x \in Q$. Aplicando o lema 2.2.4 com $p_{\varepsilon} \circ q^{-1}$ no lugar de $g$, tem-se que $\left(^{*}\right)$ é verdade. 


\section{Apêndice}

Lema A.1 Seja $X=|K|$ um poliedro finito conexo e $A$ um subpoliedro tal que, $X-A$ não tem ponto de corte local e A pode ser "by-passed". Suponha que $f:(X, A) \rightarrow(X, A)$ tem apenas um número finito de pontos fixos sobre $X-A$ e todos estão em simplexos maximais de $|K|$. Seja $p$ um caminho em $|K|$ de $x_{0}$ para $x_{1}$, onde $x_{0} \in X-A$ e $x_{0}, x_{1} \in \operatorname{Fix}(f)$.

(a) Se $x_{1} \neq x_{0}$ e $x_{1} \in|K|-A$, então $p$ é homotópico à $q$ tal que $q(I)$ é arco $P L$-normal em $(|K|, A), q(I) \cap A=\emptyset$ e $\operatorname{Fix}(f) \cap Q=\left\{x_{0}, x_{1}\right\}$.

(b) Se $x_{1} \in \operatorname{Bd} A$, então p é homotópico à $q$ tal que $q(I)$ é um arco $P L$-normal em $(|K|, A), q(I) \cap A=\left\{x_{1}\right\}$ e $\operatorname{Fix}(f) \cap Q=\left\{x_{0}, x_{1}\right\}$.

Prova: (a) Como $A$ pode ser by-passed existe um caminho $p_{1}$ em $X-A$ de $x_{0}$ para $x_{1}$ homotópico a $p$. Nós desejamos construir um caminho $q$ de $x_{0}$ para $x_{1}$ que consiste de segmentos de reta cujo interior de cada segmento esta em simplexos maximais de $|K|-A$ e os pontos finais dos segmentos estão em simplexos de $|K|-A$ de dimensão pelo menos $1, q(I) \cap \operatorname{Fix}(f)=\left\{x_{0}, x_{1}\right\}$, $q \simeq p_{1}$ rel $\{0,1\}$ e sem auto intersecção. Existe $t_{1}>0$ tal que $q_{1}=\left\{p_{1}(t) \mid 0 \leq\right.$ $\left.t \leq t_{1}\right\}$ está no fecho do simplexo maximal $\left|s_{1}\right| \subset|K|-A$, onde $x_{0} \in\left|s_{1}\right| \mathrm{e}$ $p_{1}\left(t_{1}\right) \in \mathrm{Bd}\left|s_{1}\right|$. Existe um segmento de reta $d_{1}(t), 0 \leq t \leq t_{1}$, começando em $x_{0}$ tal que $\operatorname{Fix}(f) \cap d_{1}=\left\{x_{0}\right\}, d_{1}(t) \in\left|s_{1}\right|$ para todo $0 \leq t<t_{1}, \sigma\left(d_{1}\left(t_{1}\right)\right)$ é de dimensão pelo menos 1 e $p_{1}\left(t_{1}\right) \in \overline{\left|\sigma\left(d_{1}\left(t_{1}\right)\right)\right|}$. Existe $t_{2}>t_{1}$ e um simplexo 
maximal $\left|s_{2}\right| \subset|K|-A$ tal que

$$
q_{2}=\left\{p_{1}(t) \mid t_{1} \leq t \leq t_{2}\right\} \subseteq \widehat{s_{2} \mid}
$$

e $p_{1}\left(t_{2}\right) \in \mathrm{Bd}\left|s_{2}\right|$. Se $d_{1}\left(t_{1}\right) \in \overline{\left|s_{2}\right|}$ então existe um segmento de reta $d_{2}(t), t_{1} \leq$ $t \leq t_{2}$, começando em $d_{1}\left(t_{1}\right)$, disjunto de $\operatorname{Fix}(f)$; e tal que $d_{2}(t) \in\left|s_{2}\right|$ para $t_{1}<t<t_{2}$, onde $\sigma\left(d_{2}\left(t_{2}\right)\right)$ é dimensão pelo menos 1 , e $p_{1}\left(t_{2}\right) \in \overline{\left|\sigma\left(d_{2}\left(t_{2}\right)\right)\right|}$. No entanto, se $\sigma\left(p_{1}\left(t_{1}\right)\right)$ é um vértice de $K$, então não é necessariamente verdade que $d_{1}\left(t_{1}\right) \in \overline{\left|s_{2}\right|}$. Como $|K|-A$ não ponto de corte local, existem simplexos maximais $\left|s_{2}^{1}\right|, \ldots,\left|s_{2}^{r}\right|$ de $|K|-A$ em $\left|S t\left(\sigma\left(p_{1}\left(t_{1}\right)\right)\right)\right| \operatorname{com} d_{1}\left(t_{1}\right) \in \overline{\left|s_{2}^{1}\right|}$ e $s_{2}^{i} \cap s_{2}^{i+1}$ de dimensão pelo menos 1 para $i=1, \ldots, r-1$ e $s_{2}^{r}=s_{2}$. Portanto podemos neste caso, achar um caminho $d_{2}(t), t_{1} \leq t \leq t_{2}$, começando em $d_{1}\left(t_{1}\right)$; constituido de segmentos de reta de tal forma que, exceto os pontos finais de cada segmento, cada ponto esta em simplexos maximais de $|K|-A$, e os pontos finais dos segmentos estão em simplexos de dimensão pelo menos $1 ; d_{2} \cap \operatorname{Fix}(f)=\emptyset$, e $\overline{\left|\sigma\left(d_{2}\left(t_{2}\right)\right)\right|}$ contém $p_{1}\left(t_{2}\right)$. Continuando desta maneira obtemos um caminho $q^{\prime}=d_{1} \cup d_{2} \cup \ldots \cup d_{k} \operatorname{com} q^{\prime} \simeq p_{1} \operatorname{rel}\{0,1\}$, pois cada $d_{i}$ é homotópico a $q_{i}$ pelas homotopias retas e estas homotopias são iguais nos pontos finais (ver figura 2.3).

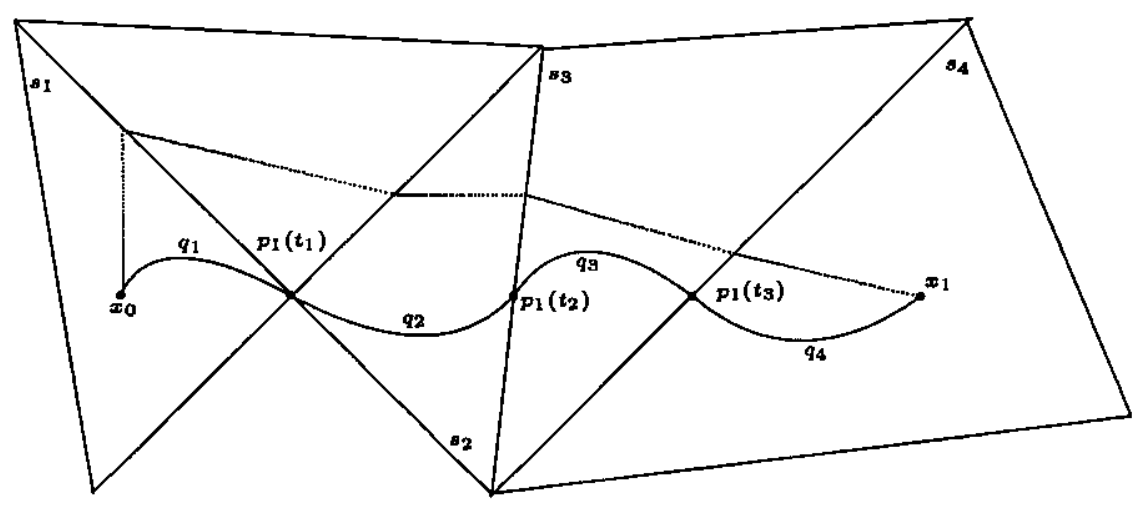

Figura 2.3: 
Note que não podemos garantir que o caminho $q^{\prime}$ não tenha auto intersecção. Para eliminar as auto interseções procedemos da seguinte forma. Seja $q^{\prime}\left(s^{\prime}\right)=q^{\prime}(s), s^{\prime}<s$, o último ponto de auto interseç̧ão de $q^{\prime}$. Façamos a seguinte construção sobre $q^{\prime}[s, 1]$ : Seja $a_{0}=q^{\prime}(s), a_{1}, \ldots, a_{n}=q^{\prime}(1)$ os cantos de $q^{\prime}[s, 1]$. Para todo $i$, trace um segmento de reta $l_{i}$ em $\sigma\left(a_{i}\right)$, de comprimento $\delta>0$, centrado em $a_{i}$, tal que $l_{0}$ esteja ao longo de $q^{\prime}\left(s^{\prime}-\delta, s^{\prime}+\delta\right)$ e $l_{n}$ seja transversal à $\left[a_{n-1}, a_{n}\right]$. Se $i \neq 0, n$ e $\sigma\left(a_{i}\right)$ é maximal, tomamos $l_{i}$ sobre a bissetriz do ângulo entre $\left[a_{i-1}, a_{i}\right]$ e $\left[a_{i}, a_{i+1}\right]$. Para todo $i$, considere os segmentos $l_{i}^{\prime}$ e $l_{i}^{\prime \prime}$ centrado no ponto médio $b_{i}$ de $\left[a_{i}, a_{i+1}\right]$, paralelo e igual à $l_{i}$ e $l_{i+1}$ respectivamente. Seja $P_{i}^{\prime}$ o paralelogramo que tem $l_{i}$ e $l_{i}^{\prime}$ como lados opostos, e $P_{i}^{\prime \prime}$ o paralelogramo que tem $l_{i}^{\prime \prime}$ e $l_{i+1}$ como lados opostos. Seja $T_{i}$ os dois triângulos ou um triângulo conectando $P_{i}^{\prime}$ e $P_{i}^{\prime \prime}$, de acordo com $P_{i}^{\prime}$ e $P_{i}^{\prime \prime}$ estão em planos diferentes ou no mesmo plano (ver figura 2.4). Coloque $P_{i}=P_{i}^{\prime} \cup T_{i} \cup P_{i}^{\prime \prime}$ e $\sum_{\delta}=\bigcup_{i=0}^{n-1} P_{i}$.
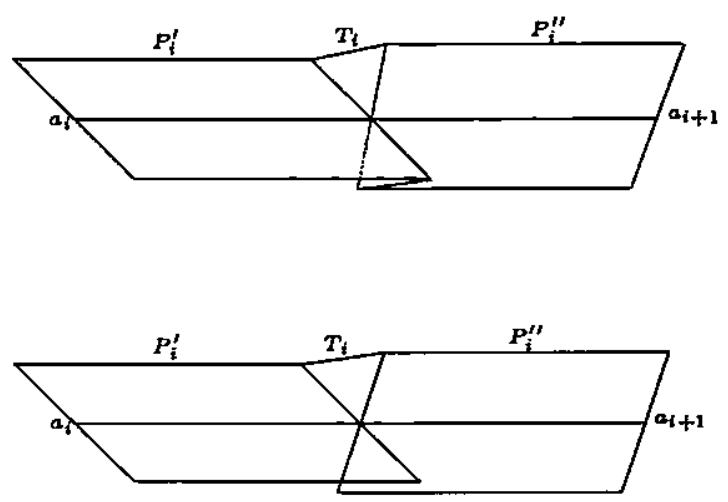

Figura 2.4:

Tome $\delta$ suficientemente pequeno de tal forma que $\sum_{\delta}$ encontre $q^{\prime}[0, s]$ somente sobre $l_{0}$ e $f$ só tenha $x_{1}$ como ponto fixo em $\sum_{\delta}$. Como existe um homeomorfismo $\eta: S \rightarrow \sum_{\delta} \subset|K|-A$, onde $S \subset \mathbb{R}^{2}$ è uma faixa retangular, que aplica o eixo de $S$ (ver figura 2.5) no arco $q^{\prime}[s, 1]$, podemos deformar o 
arco $l_{0}=\eta[a, b]$ no arco $\eta[a, c, d, b]$ sobre a faixa $\sum_{\delta}=\eta(S)$ e substituindo $l_{0}$ por $\eta[a, b, d, b]$ movemos a auto intersecção para o ponto $q^{\prime}(1)$; podemos além disso deformar este arco de tal forma que ele fique fora de $q^{\prime}[0,1]$, pois $q^{\prime}(1)$ está em um simplexo maximal. Repetindo este processo várias vezes se necessário obtemos o arco PL-normal $q$ desejado.

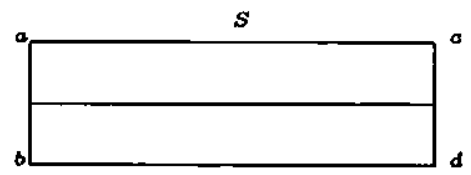

Figura 2.5:

(b) Se $x_{1} \in \operatorname{Bd} A$, então escolhemos $y \in|K|-A$ próximo de $x_{1}$ de tal forma que existe um caminho $v: I \rightarrow|K|$ de $x_{1}$ para $y \operatorname{com} v(I) \cap A=\left\{x_{1}\right\}$. Então o caminho justaposto $p v$ de $x_{0}$ para $y$ é homotópico a algum caminho $p_{1}$ em $|K|-A$. Aplicando a construção acima para o caminho $p_{1} v^{-1}$ obtemos um caminho $q$ homotópico à $p_{1} v^{-1}$ e consequentemente homotópico a $p$ que satisfaz as condições exigidas em (b) (ver figura 2.6).

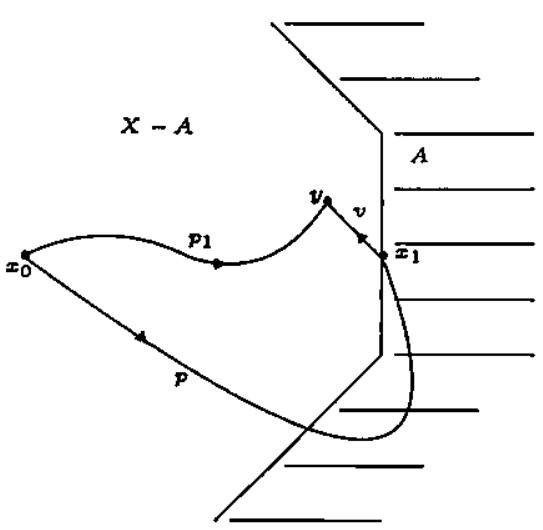

Figura 2.6: 
Lema A.2 Se adicionarmos ao lema $A .1$ a hipótese de $X-A$ não ser 2variedade, podemos obter um arco Pl-normal $p^{\prime}$ que além de satisfazer $(a) e$ (b) também satisfaz as condiçẽs $(\alpha)$ e $(\beta)$ do lema 2.2.6.

Prova: O fato de $X-A$ não ser 2-variedade, implica que para alguma triangulação $K$ de $X$ temos um 1-simplexo $\left|\sigma_{1}\right| \subset|K|-A$ sendo face de pelo menos três 2-simplexos $|\sigma|,\left|\sigma^{\prime}\right|$ e $\left|\sigma^{\prime \prime}\right|$ de $|K|-A$. Podemos supor que $\left|\sigma_{1}\right|$ é face comum de pelo menos dois simplexos maximais em $|K|-A$, caso contrário usamos alguma subdivisão de $K$ ao invés de $K$.

Considere o arco PL-normal $q$ construído no lema A.1. Podemos ligar um ponto $a_{1}$ de $q[0,1]$ que não é canto, por segmentos de reta que não contém pontos fixos de $f$, para algum ponto $c_{1}$ de $\left|\sigma_{1}\right|$ tal que; este segmento intercepta $q[0,1]$ somente em $a_{1}$. Repetimos este processo com pontos $b_{1} \in q[0,1]$ e $d_{1} \in\left|\sigma_{1}\right|$ (ver figura 2.7). Tome um pequeno simplexo $|\tau|$ em $|\sigma|$ de mesma dimensão de tal forma que uma de suas faces esteja contida em $\left|\sigma_{1}\right|$ e contenha $\left[c_{1}, d_{1}\right]$. Desenhe um ponto $e$ em $|\tau|$. O caminho $p^{\prime}$ desejado será o arco PL-normal que vai de $x_{0}$ para $a_{1}$ ao longo de $q$, então ao longo de $\left[a_{1}, \ldots, c_{1}, e, d_{1}, \ldots, b_{1}\right]$, e então de $b_{1}$ para $x_{1}$ ao longo de $q$.

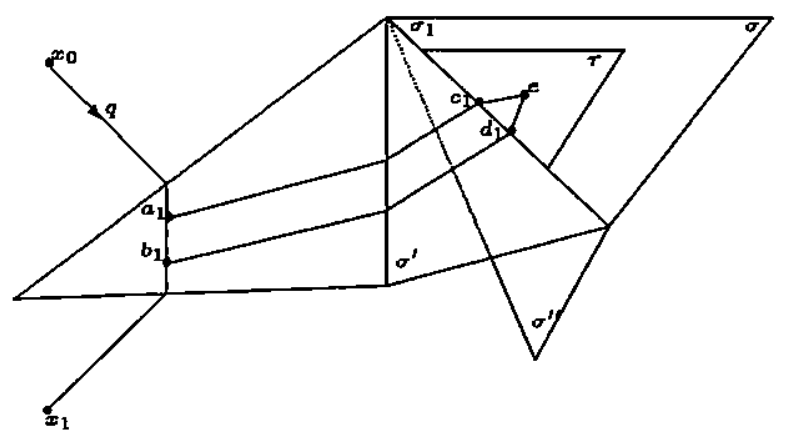

Figura 2.7: 
Lema A.3 Seja $|K|$ um poliedro finito conexo sem ponto de corte local. Todo caminho especial $p: I \rightarrow|K|$ é especialmente homotópico a algum caminho PL-normal $p^{\prime}$.

Prova: Seja $q$ o caminho em relação o qual $p$ é especial e $q(0)=p(0)=a$, $q(1)=p(1)=b$. Eles são dois pontos diferentes e não são vértices de $K$, pois $q$ é por hipótese normal. Tome $\varepsilon>0$ tal que $q$ é linear sobre $[0, \varepsilon]$ e $[1-\varepsilon, 1], p[0, \varepsilon] \subset|S t(\sigma(a))|$ e $p[1-\varepsilon, 1] \subset|S t(\sigma(b))|$. Construa uma homotopia $h: I \times I \rightarrow X$ da seguinte forma. Defina $h$ sobre $[0, \varepsilon] \times I$ por

$$
h(s, t)= \begin{cases}\left(1-\frac{s}{\varepsilon t}\right) a+\frac{s}{\varepsilon t} p(\varepsilon t) & \text { se } s \leq \varepsilon t \text { e } t>0, \\ p(s) & \text { se } s \geq \varepsilon t .\end{cases}
$$

Temos $h(s, t) \neq q(s)$ para $0<s \leq \varepsilon$, pois como $q$ é linear sobre $[0, \varepsilon]$ tem-se

$$
q(s)=\left(1-\frac{s}{\varepsilon t}\right) a+\frac{s}{\varepsilon t} q(\varepsilon t) .
$$

Defina $h$ sobre $[1-\varepsilon, 1] \times I$ de maneira similar, isto é,

$$
h(s, t)= \begin{cases}\left(1+\frac{s-1}{\varepsilon t}\right) b+\left(\frac{-s+1}{\varepsilon t}\right) p(1-\varepsilon t) & \text { se } s \geq 1-\varepsilon t \text { e } t>0, \\ p(s) & \text { se } s \leq 1-t \varepsilon .\end{cases}
$$

Agora

$$
\eta=\inf \{d(p(s), q(s)) \mid s \in[\varepsilon, 1-\varepsilon]\}>0 .
$$

Como $|K|$ não tem ponto de corte local, por uma construção semelhante a feita no lema A.1 parte (a), podemos obter um caminho PL-normal que é $\eta$-homotópico à $p[\varepsilon, 1-\varepsilon]$. Seja $h:[\varepsilon, 1-\varepsilon] \times I \rightarrow|K|$ a $\eta$-homotopia, então 
$h(\varepsilon, t)=p(\varepsilon), h(1-\varepsilon, t)=p(1-\varepsilon), h(s, 0)=p(s), h(s, t) \neq q(s)$ para todo $s \in[\varepsilon, 1-\varepsilon]$ e $t \in I$, pois $\operatorname{diam}\{h(s, t) ; 0 \leq t \leq 1\}<\eta$. Colando as três partes, nós chegamos a uma homotopia especial $h: I \times I \rightarrow X$, com $h_{1}$ um caminho PL. Mudando os cantos de $h_{1}$ se necessário, chegamos ao caminho PL-normal desejado $p^{\prime}$.

Lema A.4 Considere $|K|$ um poliedro finito conexo sem ponto de corte local e $q$ um arco $P L$-normal satisfazendo a condição $(\alpha)$ do lema 2.2.6. Então, qualquer caminho especial $p: I \rightarrow|K|$ é especialmente homotópico a um caminho $p^{\prime}: I \rightarrow|K|$ com $p^{\prime}[0,1] \cap(q(0,1) \cup \tau)=\emptyset$.

Prova: Podemos supor que $p$ é um caminho PL-normal pelo lema A.3. Mudando os cantos se necessário, podemos supor que os cantos de $p$ não estão em $Q$, e consequentemente as intersecções de $p$ e $Q$ é um número finito.

Eliminaremos as intersecções do seguinte modo. Seja $p\left(s^{\prime}\right)=q(s)$ uma intersecção. Se $s^{\prime}<s$, fazemos a construção do lema A.1 parte (a) sobre o arco $q[s, 1]$ com $l_{0}$ ao longo de $p\left(s^{\prime}-\delta, s^{\prime}+\delta\right)$, podemos deformar especialmente uma pequena parte de $p$ em torno de $s^{\prime}$ de tal forma que a intersecção move-se ao longo de $Q$ para $q(1)$ e fora de $q(0,1)$. O caso $s^{\prime}>s$ pode ser tratado de forma similar, mas a construção é feita sobre $q[0, s]$. Portanto repetindo este processo várias vezes se necessário obtemos um caminho PLnormal $p_{1}$, especialmente homotópico a $p$, tal que $p_{1}[0,1] \cap q(0,1)=\emptyset$.

Empurre $p_{1}(I) \cap \tau$ para o bordo de $\tau$ pela projeção radial de $q\left(s_{3}\right) \in \tau$. A condição $(\alpha)$ é necessária para evitar a intersecção com $Q$ durante o empurrão. Assim $p^{\prime}$ é o caminho desejado. 
Lema A.5 Suponha que o arco PL-normal $q$ satisfaça $(\alpha)$ do lema 2.2.6. Seja $p_{0}, p_{1}: I \rightarrow|K|$ dois caminhos especiais tal que as imagens $p_{0}(I) e$ $p_{1}(I)$ não interceptam $q(0,1) \cup \tau$. Se $p_{0}$ e $p_{1}$ são homotópicos em $|K|-\tau$, então eles são especialmente homotópicos em $|K|$.

Prova: Seja $\lambda: I \rightarrow I$ definido da forma

$$
\lambda(s)= \begin{cases}0, & \text { se } 0 \leq s \leq s_{1} \\ \frac{s-s_{1}}{s_{2}-s_{1}}, & \text { se } s_{1} \leq s \leq s_{2} \\ 1, & \text { se } s_{2} \leq s \leq s_{1}\end{cases}
$$

Os caminhos $p_{0}$ e $p_{0} \circ \lambda$ são ligados pela homotopia $h: I \times I \rightarrow|K|$ definida por

$$
h(t, s)=p_{0}((1-t) s+t \lambda(s))
$$

que é fácil ver ser especial. Similarmente $p_{1}$ e $p_{1} \circ \lambda$ são especialmente homotópicas. Seja $p: I \times I \rightarrow|K|-\tau$ uma homotopia ligando $p_{0}$ e $p_{1}$. Então $\left\{p_{t} \circ \lambda\right\}_{t \in I}$ é uma homotopia ligando $p_{0} \circ \lambda$ e $p_{1} \circ \lambda$. Esta é especial, pois $p_{t} \circ \lambda(s)=q(0) \neq q(s)$ sobre $\left(0, s_{1}\right], p_{t} \circ \lambda(s)=q(1) \neq q(s)$ sobre $\left[s_{2}, 1\right)$, e sobre $\left(s_{1}, s_{2}\right)$ nós temos $p_{t} \circ \lambda(s) \in|K|-\tau$ mas $q(s) \in \tau$.

Lema A.6 Seja $|K|$ um poliedro finito conexo sem ponto de corte local e $q$ um arco $P L$-normal que satisfaz as condições $(\alpha)$ e $(\beta)$ do lema 2.2.6. Se dois caminhos especiais $p_{0}, p_{1}: I \rightarrow|K|$ são homotópicos, então eles são especialmente homotópicos.

Prova: Em vista do lema A.4, podemos supor que $p_{0}[0,1]$ e $p_{1}[0,1]$ são disjuntos de $q(0,1) \cap \tau$. Escolha o ponto $a=q(0)$ como ponto base de $|K|$ e $|K|-\tau$. $p_{0} \simeq p_{1} \operatorname{rel}\{0,1\}$ implica que $p_{1} p_{0}^{-1} \simeq 1 \operatorname{rel}\{0,1\}$ em $|K|$ (onde 1 
representa o caminho constante).

Caso 1: $\operatorname{dim} \tau \geq 3$. A inclusão $i:|K|-\tau \subset|K|$ induz um isomorfismo $i_{*}: \pi_{1}(|K|-\tau) \rightarrow \pi_{1}(|K|)$. Agora $p_{0} \simeq p_{1} \operatorname{rel}\{0,1\}$ em $|K|$ se e somente se $p_{0} \simeq p_{1} \operatorname{rel}\{0,1\}$ em $|K|-\tau$, e pelo lema $A .5, p_{0}$ e $p_{1}$ são especialmente homotópicos.

Caso 2: $\operatorname{dim} \tau=2$. Tome um ponto $c$ sobre $\mathrm{Bd} \tau$ que não é vértice de $K \mathrm{e}$ é diferente de $q\left(s_{1}\right)$ e $q\left(s_{2}\right)$, seja $u$ o laço sobre $\mathrm{Bd} \tau$, com ponto base em $c$ e que dá uma volta em torno de Bd $\tau$. Pelo Teorema de Van Kampen $\pi_{1}(|K|)$ é isomorfo ao grupo quociente de $\pi_{1}(|K|-\tau)$ módulo o subgrupo normal gerado por $u$. Como uma consequência de $p_{1} p_{0}^{-1} \simeq 1 \mathrm{rel}\{0,1\}$ em $|K|, p_{1} p_{0}^{-1}$ é homotópico em $|K|-\tau$ a um laço da forma $\left(v_{1} u^{k_{1}} v_{1}^{-1}\right)\left(v_{2} u^{k_{2}} v_{2}^{-1}\right) \ldots\left(v_{m} u^{k_{m}} v_{m}^{-1}\right)$, onde $k_{1}, \ldots, k_{m}$ são inteiros, $v_{1}, \ldots, v_{m}$ são caminhos em $|K|-\tau$ de $a$ para $c$.

Para todo $j=1, \ldots, m$ podemos achar um caminho PL $v_{j}^{\prime}$, tendo somente um número finito de interseç̧ões com $Q$, tal que $v_{j}^{\prime} \simeq v_{j} \operatorname{rel}\{0,1\}$ em $|K|-\tau$. Então eliminamos as intersecções de $v_{j}^{\prime}$ com $q(0,1)$ exatamente como no lema A.4. Assim obtemos um caminho $w_{j}$ em $|K|-(q(0,1) \cup \tau)$ tal que $w_{j} \simeq v_{j} \operatorname{rel}\{0,1\}$ em $|K|-\tau$.

Tome uma vizinhança $W$ de $a$ tal que $\bar{W} \cap \bar{\tau}=\emptyset$. A condição $(\beta)$ torna possível modificar $u$ em um laço $\mu \mathrm{em}|K|-(q(0,1) \cup \tau)$, com ponto base em $c$ e tal que $\mu \simeq u$ rel $\{0,1\}$ em $|K|-\tau-W$. A figura 2.8 mostra como isto é feito.

Seja $p_{0}^{\prime}=\left(w_{1} \mu^{k_{1}} w_{1}^{-1}\right) \ldots\left(w_{m} \mu^{k_{m}} w_{m}^{-1}\right) p_{0}$. Agora $p_{1}$ e $p_{0}^{\prime}$ estão em $|K|-$ $(q(0,1) \cup \tau)$, e eles são homotópicos em $|K|-\tau$. Pelo lema A.5 $p_{1}$ e $p_{0}^{\prime}$ são especialmente homotópicos. Resta mostrar que $p_{0}$ e $p_{0}^{\prime}$ são especialmente homotópicos.

Seja $\varepsilon>0$ tal que $q[0, \varepsilon]$ está contida na vizinhança $W$ de $a$ escolhida acima. Em vista das técnicas de reparametrização usada na demonstração 


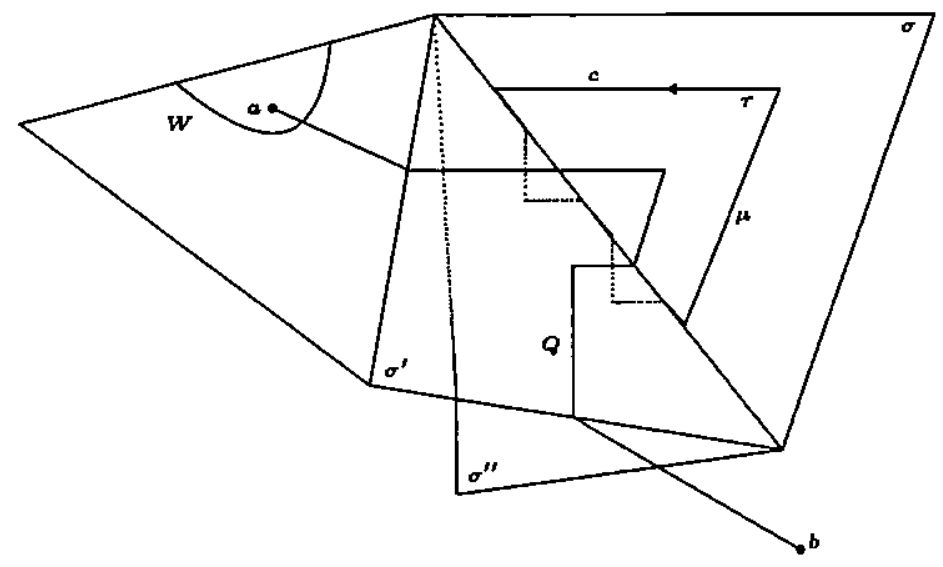

Figura 2.8:

do lema A.5, podemos supor que $p_{0}$ é constante sobre $[0, \varepsilon]$, e que $p_{0}^{\prime}$ é igual $\left(w_{1} \mu^{k_{1}} w_{1}^{-1}\right) \ldots\left(w_{m} \mu^{k_{m}} w_{m}^{-1}\right)$ sobre $[0, \varepsilon]$ e igual à $p_{0}$ sobre $[\varepsilon, 1]$. Como $\mu \simeq$ $u \operatorname{rel}\{0,1\}$ em $|K|-\tau-W$ mas $u \simeq 1 \operatorname{rel}\{0,1\}$ em $\bar{\tau} \subset|K|-W, \mu \simeq$ 1 rel $\{0,1\}$ em $|K|-W \subset|K|-q(0, \varepsilon]$. Agora $w_{j}$ está em $|K|-q(0,1) \subset$ $|K|-q(0, \varepsilon]$, deste modo $\left(w_{j} \mu^{k_{j}} w_{j}^{-1}\right) \simeq w_{j} w_{j}^{-1} \operatorname{rel}\{0,1\}$ e $w_{j} w_{j}^{-1} \simeq 1 \operatorname{rel}\{0,1\}$ em $|K|-q(0, \varepsilon]$. Daí , a parte de $p_{0}^{\prime}$ sobre $[0, \varepsilon]$ pode ser deformada a uma constante sem tocar $q(0, \varepsilon]$. Assim $p_{0}$ e $p_{0}^{\prime}$ são especialmente homotópicas. 


\section{Referências Bibliográficas}

[1] Helga Schirmer, A relative Nielsen number, Pacific J. Math., 122 No.2 (1986), 459-473.

[2] Boju Jiang, On the least number of fixed points, Amer. J. Math., 102 No.4 (1980), 749-763.

[3] Boju Jiang, Lectures on Nielsen Fixed Point Theory, Contemporary Mathematics v.14, Amer. Math. Society, Providence, Rhode Island, 1983.

[4] Xuezhi Zhao, On minimal fixed point numbers of relative maps, preprint, Department of Mathematics, Peking University, China.

[5] Robert F. Brown, The Lefschetz Fixed Point Theorem, Scott, Foresman and Co., Glenview, Il., 1971.

[6] Kiang Tsai-han, The Theory of Fixed Point Classes, Springer-Verlag, 1989.

[7] E. H. Spanier, Algebraic Topology, McGraw-Hill, New York, 1966.

[8] D. L. Gonçalves e J. C. de Souza Kiihl, Teoria do Índice, $14^{\circ}$ Colóquio Brasileiro de Matemática - IMPA, Poços de Caldas, 1983. 